\title{
Circular Scale of Time and Construction of the Schrödinger Perturbation Series for Energy Made Simple
}

\author{
Stanisław Olszewski \\ Institute of Physical Chemistry, Polish Academy of Sciences, Warsaw, Poland \\ Email: olsz@ichf.edu.pl
}

How to cite this paper: Olszewski, S. (2017) Circular Scale of Time and Construction of the Schrödinger Perturbation Series for Energy Made Simple. Journal of Modern Physics, 8, 1650-1684. https://doi.org/10.4236/jmp.2017.89098

Received: July 25, 2017

Accepted: August 28, 2017

Published: August 31, 2017

Copyright $\odot 2017$ by author and Scientific Research Publishing Inc. This work is licensed under the Creative Commons Attribution International License (CC BY 4.0).

http://creativecommons.org/licenses/by/4.0/

\section{(c) (i) Open Access}

\begin{abstract}
Physically the examined perturbation problem can be regarded as a set of collision events of a time-independent perturbation potential with a quantum system. As an effect of collisions there is an expected definite change of energy of an initially unperturbed state of the system to some stationary perturbed state. The collision process certainly occupies some intervals of time which, however, do not enter the formalism. A striking property is the result of a choice of the sequence of collisions according to the applied circular scale of time: the scale produces almost automatically the energy terms predicted by the Schrödinger perturbation theory which usually is attained in virtue of complicated mathematical transformations. Beyond of the time scale and its rules-strictly connected with the perturbation order $N$ introduced by Schrödinger-a partition process of the number $N-1$ is applied. This process, combined with contractions of the time points on the scale, provides us precisely with the perturbation terms entering the Schrödinger theory.
\end{abstract}

\section{Keywords}

Circular Scale of Time, Schrödinger Perturbation Series for Energy, Non-Degenerate Quantum State

\section{Introduction}

The present paper has two aims. The first one is to provide an evident simplification of the treatment of the Schrödinger perturbation series for energy, especially at large perturbation order $N$. Another aim is to demonstrate that a circular scale of time can be indispensable in realization of the first aim.

According to the definition of the German "Physikalisches Wörterbuch" [1], 
time is an independent variable in the classical mechanics. In fact the description of any motion cannot be done without the use of the notion of time: all fundamental mechanical parameters like the position change of a body, its velocity, acceleration, the force acting in course of the motion, apply the notion of time. With the development of the theory of relativity, a special attention has been attached to the time interval and its peculiar properties.

A special treatment concerning the time notion is applied in the quantum theory; see e.g. [2] [3]. Here the time variable does not possess its independent operator, so only the changes of the observables obtained with a change of time are considered. This kind of approach is done on the basis of commutators of the operators representing the observables with the Hamiltonian operator. In fact the energy operator becomes a central operator and energy variable becomes a central physical parameter of the quantum formalism. A characteristic point is that the notion of the time interval is practically banned out of the quantum theory in which a probabilistic approach is mainly applied in description of the effects connected with the particle transitions.

A general idea of the present and former papers by the author [4]-[9] is to point out the time importance in the use of the quantum perturbation theory. This theory has been developed [10] simultaneously with the Schrödinger wave-mechanical approach to the quantum systems [11] [12] [13]. Evidently it became clear already at the introduction moment of the Schrödinger wave mechanics that an exact application of that theory is possible only for very special physical systems. This means that in a treatment of other systems only the approximate solutions should be sought and used. Typical situations concerned an interest in the systems which differ only slightly from the systems which are easy to solve. The difference could be reduced to a rather small potential present in a more complicated Hamiltonian. Such a potential-called the perturbation potential-when combined with the wave functions and energies of a more simple system, could provide us with an approach to similar parameters of a more complicated system. The corresponding formalism-elaborated by Schrödinger and called the perturbation theory [10] - became a purely mathematical problem in solving of which the notion of time has not been applied. Nevertheless, an accurate treatment of the perturbation formalism occurred to be an extremely complicated task. This difficulty becomes clear already at the stage of presentation of the problem.

But in a series of former papers [4]-[9] an attempt has been done to demonstrate that the introduction of the time notion, as well as application of the circular scale of time as an appropriate scale in the perturbation theory, can substantially simplify that theory and its calculations. The aim of the present paper is to outline the perturbation theory in a still more simple way.

In Section 2 we present the properties of the time scale which are essential for its application. It should be noted that the time variable and the Feynman diagrams based on it have been used a long time before the present approach to 
the Schrödinger perturbation theory has been developed [14]. The results and drawbacks of the method based on the Feynman diagrams are discussed in Section 3.

\section{Scale of Time Considered in Reference to the Properties of the Observed System as Well as Abilities of the Observer}

Time is an evidently subjective notion because it depends on the physical phenomena represented by the observed system, as well as abilities possessed by an observer. In reality the physical and philosophical properties of the notion of time were combined gradually with the experience and observation of the everyday life; science-excepting perhaps for astronomy-had, at least at its early stage, not much to do with time. A separate component of the view on time is provided by the human imagination. This second component seems to be mainly responsible for application of the time notion-with a variable degree of certainty-from the atom to universe.

It is easy to demonstrate a subjective character of time mentioned above. If we limit our "universe" to one hydrogen atom, and the observer's ability to distinction between the atomic nucleus and electron as well as the size of the distance separating these both objects, we obtain two possibilities concerning time. The first one-created by assuming a constant nucleus-electron distance in course of the electron motion done, say, along a circle-cannot serve to establish any notion of time because no change of the system can be detected by the observer. But another situation is obtained when the distance between two mentioned particles changes systematically, say in effect of a planar motion of the electron along an ellipse. In this case the observer's measurements are spread within the interval length equal to a double difference between the longer and shorter semi axis of the ellipse. If the motion is perfectly periodic, the observed interval of length repeats after the same period of time $T$. In result all time points accessible by the observations are enclosed within the interval

which repeats incessantly because no limit is imposed on the electron motion along the ellipse.

However, the everyday observations on time are evidently against the limit given in (1). The effect of these observations combined with imagination imposes a replacement of $T$ in (1) by infinity:

$$
(0, \infty) \text {. }
$$

Moreover, a further analysis of the contemporary situation as an effect of earlier situations combined again with imagination, provides us also with an infinite size of the interval of time concerning the past. In effect this gives a commonly admitted interval

$$
(-\infty, \infty) \text {. }
$$

Characteristically, the interval (3) encloses practically all possible events in 
nature, but it does not explain much what happens within (2) or (3). A rather simple example can be suggested by the quantum theory.

In the modern theory of atom-an object which is best penetrated by the quantum physics-we have a positively charged nucleus surrounded by the cloud of a negative electron charge. If the atom is in its lowest state of energy, called also the ground state, and no external fields or collisions act on it, it can remain-according to the present knowledge-in such a state practically infinitely long time with no change. Therefore no idea, or scale, of time can or should be applied in order to describe such atom.

But a different situation is obtained when-at some moment-the atom becomes perturbed, for example by the action of an external field. Let us assume that this field is independent of time. If the time moment of inclusion of the perturbation potential is denoted by

$$
t=t_{1}=0
$$

in any time moment

$$
t_{2}>t_{1}
$$

the properties of the atom are changed in comparison with those possessed at time (4). However-for a sufficiently weak perturbation potential $V^{\text {per }}$-we can assume that in effect of the action of $V^{\text {per }}$ at some

$$
t=t_{l} \gg 0
$$

the atom will approach another stationary state, certainly different than that occupied at $t=0$. The stationary state means that at

$$
t \gg t_{l}
$$

the atom properties will be not effectively different than those possessed at $t=t_{l}$. In other words the atom behaves at (7) as an unchanged object identical to that obtained at time $t=t_{l}$ in (6). For such an object the idea of time loses its sense. A question becomes how time is going within the interval between $t=0$ and $t=t_{l}$.

\section{Feynman's Treatment and Present Treatment of the Time Interval $\left(0, t_{l}\right)$ Characteristic for the Perturbation Process}

A fundamental difference in the Feynman's and present treatment of the Schrödinger perturbation series concerns the system behaviour in dependence on time. In fact Feynman assumes that the time interval followed by a physical system on his diagrams is of an unlimited length, i.e. the interval is that of (2) or (3); see [15]. The answer of the atomic system to an applied perturbation is a set of energy transitions in different time moments done to different quantum states which are possible to be attained for an unperturbed atom. If the degeneracy with respect to energy of the system does not hold, only one of the system states is the ground state, the other ones are necessarily the excited states. In principle all excited states of the unperturbed system are admissible for the energy transitions 
in the perturbation process. In order to obtain a final result of the perturbation, the Feynman's method admits that gradually more and more time moments are involved in the system contacts with the perturbation potential.

But these contacts are arranged practically on an equal footing: there does not exist a classification of the time moments which are more or less admissible by the system in course of its way with time. In effect a convergent result for the perturbation energy requires an enormous number of diagrams, or energy components, in order to approach a final perturbation result. This is expected to hold especially when the physical nature of the system makes the perturbation series only slowly convergent in the original, i.e. time-independent, Schrödinger perturbation formalism.

A look on the original approach proposed by Schrödinger makes it clear that the quantum system should contact its perturbation in different but specified ways, in dependence on the number of contacts. A full number of contacts of a given kind were called the perturbation order labeled by $N$; an increasing number of considered orders evidently increase the accuracy of a final perturbation result. Mathematically this led to special perturbation terms the number of which was strictly connected with the order $N$. When the perturbation of a non-degenerate quantum system was considered, the number of the perturbation terms $S_{N}$ characteristic for a given $N$ could be expressed by the formula [16] [17]

$$
S_{N}=\frac{(2 N-2) !}{N !(N-1) !} \text {. }
$$

But the number of the Feynman diagrams $P_{N}$, classified as belonging to the order $N$ and necessary to obtain the energy contribution corresponding to $N$, was [14]

$$
P_{N}=(N-1) !
$$

For a large perturbation order, say $N=20$, we obtain from (9) the number of the Feynman terms equal to

$$
P_{20}=19 ! \approx 1.216 \times 10^{17} .
$$

On the other hand, the number of the Schrödinger perturbation terms from the formula (8) and $N=20$ becomes

$$
S_{20} \approx 1.767 \times 10^{9} \text {. }
$$

This means that in average

$$
\frac{P_{20}}{S_{20}} \cong 0.7 \times 10^{8}
$$

Feynman terms should be combined in order to give a contribution furnished by a single Schrödinger perturbation term for energy. In a computational practice this task could be difficult to be both programmed and performed.

The aim of the present method-instead to formulate a new computational problem-was a search to simplify both the scheme given by Feynman and that by Schrödinger. Since the atomic system and its perturbation potential remain 
constant, the time scale of the perturbation events became the object of an analysis. In fact we show that a suitable sequence of the system collisions with the perturbation potential done according to a circular scale of time can provide us readily with the perturbation terms given by Schrödinger and formula (8). Moreover, the individual terms of the Schrödinger series could be obtained from the proposed time scale without following their complicated derivation presented in $[10]$.

\section{The Schrödinger Perturbation Formalism and a Suitable Scale of Time}

As a first step we outline the fundamentals of the Schrödinger perturbation formalism specified for the case when a non-degenerate quantum state of a single particle is perturbed. Let this state be, for example, the lowest one of a set of non-degenerate quantum states being the eigenstates of the unperturbed and time-independent Hamiltonian operator $\hat{H}_{0}$. The unperturbed eigenenergies are

$$
E_{1}^{(0)}<E_{2}^{(0)}<E_{3}^{(0)}<E_{4}^{(0)}<\cdots
$$

so $E_{1}^{(0)}$ is the lowest energy. The time-independent parts of the eigenfunctions of $\hat{H}_{0}$ corresponding to energies (13) are

$$
\psi_{1}, \psi_{2}, \psi_{3}, \psi_{4}, \cdots
$$

so for any pair of $E_{i}$ and $\psi_{i}$ there is satisfied the eigenequation

$$
\hat{H}_{0} \psi_{i}=E_{i}^{(0)} \psi_{i} \text {. }
$$

Our aim is to calculate the eigenenrgies and eigenfunctions of a perturbed Hamiltonian

$$
\hat{H}^{\text {per }}=\hat{H}_{0}+V^{\text {per }}
$$

where

$$
\hat{V}^{\text {per }}=V^{\text {per }}(\boldsymbol{r})
$$

is a time-independent perturbation potential dependent solely on the particle position $\boldsymbol{r}$. We assume that the potential (17) is sufficiently small to represent the perturbation formalism by a convergent procedure.

In principle we seek for the wave-function solutions

$$
\psi_{1}^{\text {per }}, \psi_{2}^{\text {per }}, \psi_{3}^{\text {per }}, \cdots
$$

and eigenenergies

$$
E_{1}^{\mathrm{per}}, E_{2}^{\mathrm{per}}, E_{3}^{\mathrm{per}}, \cdots
$$

of a new eigenproblem

$$
\hat{H}^{\text {per }} \psi_{i}^{\text {per }}=E_{i}^{\text {per }} \psi_{i}^{\text {per }},
$$

but this may occur to be much more difficult than solution of an unperturbed problem (15). In general, by considering the energy alone, we look for a series 


$$
E_{1}^{\mathrm{per}}=E_{1}^{(0)}+\Delta E_{1}^{(1)}+\Delta E_{1}^{(2)}+\Delta E_{1}^{(3)}+\cdots
$$

where

$$
E_{1}^{(0)}
$$

is the unperturbed energy and

$$
\Delta E_{1}^{(i)}
$$

are the correcting terms of (22) arranged according to an increasing perturbation order

$$
i \equiv N=1,2,3,4, \cdots
$$

In general any

$$
\Delta E_{1}^{(i)}
$$

is a combination of the matrix elements

$$
\left\langle m\left|V^{\text {per }}\right| n\right\rangle
$$

where

$$
|m\rangle=\psi_{m}
$$

and

$$
|n\rangle=\psi_{n}
$$

are the unperturbed wave functions from the set (11).

Beyond of the matrix elements (26) also the unperturbed eigenenergies (13) enter the expressions (25). Excepting for the term

$$
\Delta E_{1}^{(1)}=\left\langle 1\left|V^{\text {per }}\right| 1\right\rangle,
$$

other series components $\Delta E_{1}^{(i)}$ entering the sum (21) are represented by expressions containing infinite sums over the quantum states. A single infinite sum is characteristic solely for $N=2$ :

$$
\Delta E_{1}^{(2)}=\sum_{p \neq 1} \frac{\left|\left\langle 1\left|V^{\text {per }}\right| p\right\rangle\right|^{2}}{E_{1}^{(0)}-E_{p}^{(0)}} ;
$$

here and in (29) the bra $\langle 1|$ means $\psi_{1}$ and ket $|p\rangle$ refers to any energy state beyond of that labeled by 1 .

A total number of the kinds of terms which compose the sum of

$$
\Delta E_{1}^{(i)}
$$

belonging to successive perturbation orders $i$ is equal to

$$
\sum_{i} S_{i}
$$

where $S_{i}$ is presented in (8) for $i=N$.

A difficulty is in construction of particular terms entering any (31). With the aid of the Schrödinger formalism this construction becomes a very complicated task, especially for large $i=N$. But this task can be evidently simplified by in- 
troducing the scale of time suitable for the Schrödinger perturbation problem.

\section{Properties of the Time Scale}

In general any scale of time is defined by the order in which the physical events do succeed on it. In a conventional scale of time, assumed for example in construction of the Feynman diagrams, the scale is extended from a minus to plus infinity. Therefore the time moment of the next event is compulsorily more distant from the beginning point than a former event; the events, in the perturbation theory, are represented by collisions of the perturbation potential with an unperturbed quantum system.

For the Schrödinger theory the perturbation order $i$, labeled usually by $N$, is suitable as a basis of classifying the events. For example it is convenient to assume that a separate amount of collisions belong to the order $N=1$, another set of collisions belong to $N=2$, still another set belongs to $N=3$, etc. When the scale of time for a set of collisions is assumed to be not infinitely progressive but circular, its advantage is that the Schrödinger perturbation terms occur almost automatically from it. The basic postulate is that collisions of the external perturbation potential with an unperturbed system can be grouped together into specific sets. The total number of the time points of collisions which enter such a set does not exceed $N$. So we have one collision point of time in the set belonging to $N=1$, two collision time points in the set belonging to $N=2$, three collision time points in the set for $N=3$, etc. For each set, after travelling the whole set of time points belonging to that set, the system returns to its beginning time point after which a new set of collisions can begin. This situation provides us with an unlimited number of the time scales of a circular character, each scale having $N=1,2,3, \cdots$ time points on it. For $N=1$ we have only one point on the scale which represents a beginning-end point of this scale, but the scales belonging to $N>1$ have evidently one or more time points beyond the beginning-end point of time; see Figure 1 where diagrams for $N=1,2$, and 3 are presented.

In order to obtain a full set of the Schrödinger perturbation terms from the time points belonging to the scale of a given set of $N$ points, the time points on

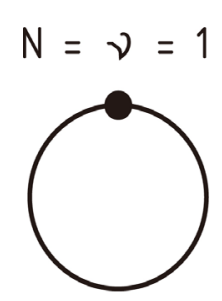

(a)

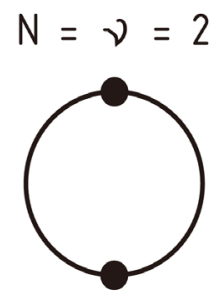

(b)

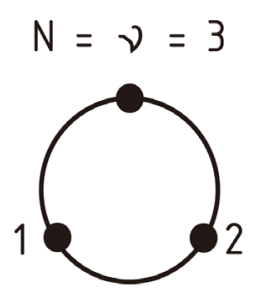

(c)

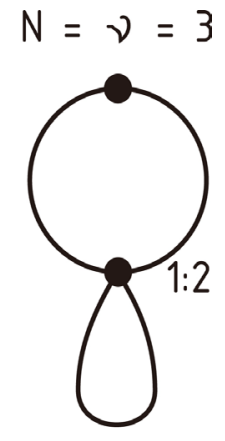

(d)

Figure 1. Diagrams denoting the Schrödinger perturbation terms for $N=1,2$ and 3. 
the scale should have a property to merge together in a definite way [see e.g. Figure 1(d)]. The fundamental rules concerning the merging process are two: (i) the beginning-end point on the scale describing a whole perturbation term is free from the merging process; (ii) the merging between two or more time points present on the scale can be done only in the way that a sequence of the time events represented by these points is not violated by the merging process.

This means, for example, that two points on the scale, say

$$
t_{a} \text { and } t_{b}, t_{c} \text { and } t_{d}, t_{e} \text { and } t_{f}, \cdots
$$

can be merged together on condition that any of these points is not a beginning-end point on the scale; moreover the points represent a sequence

$$
t_{a}<t_{b}, t_{c}<t_{d}, t_{e}<t_{f}, \cdots
$$

which is indicated by the fact that

$$
a<b, c<d, e<f, \cdots
$$

The merging symbols representing the individual Schrödinger perturbation terms given by contractions mentioned in (33) and (34) are:

$$
a: b, c: d, e: f, \cdots
$$

The merging process can be extended to a larger number of the time points than two. For example three time points $t_{a}, t_{b}, t_{c}$ can be also merged together. This situation is represented by the contraction symbols

$$
\begin{gathered}
a: b: c, \\
a: b: c: d,
\end{gathered}
$$

etc., for which the sequence relations

$$
\begin{gathered}
a<b<c, \\
a<b<c<d,
\end{gathered}
$$

are satisfied. Certainly the number of the time points participating in any merging process is limited by $N-1$ where the term -1 is due to exclusion of one, i.e. the beginning-end point of time on the scale.

But there exist also combined merging processes of the time points which can be accepted by the theory. For example

$$
a: d \frown b: c
$$

can be a valid contractions pair on condition that there holds the relation

$$
a<b<c<d
$$

which is identical with (37a).

The merging processes listed above provide us with the side loops of time which are formed on the scale together with the main loop of time. The main loop is considered to be that loop on which the beginning-end point of the scale is present. Evidently some time points lying formerly on the main loop can be shifted to a side loop, or loops, in effect of the merging process.

By considering several sets of the merged points the rule (ii) mentioned above 
should be taken into account. This means, for example, that there is not allowed a crossing of the time loops on the diagram; see e.g. [4].

A consequent application of the idea outlined above provides us with a full set of the Schrödinger perturbation terms for energy belonging to any order $N$; see Section 6 . These calculations, limited to $N=7$, have been presented already before [8] [9]. However a plain rule allowing for calculating all perturbation terms belonging to any given $N$ was lacking. In the next Sections we develop such rule on the basis of partitions of $N$ and contractions of the time points entering these partitions.

\section{Partitions of the Time Points on the Scale}

The next step to obtain the Schrödinger series is a partition of the number

$$
N-1
$$

which is the perturbation order $N$ decreased by one. The -1 is a correction term due to a circular character of the time scale. The partitions done for individual $N$ (and contractions of the time points for low $N$ ) are given in the Tables, see Tables 1-4 and Table 6.

Evidently the perturbation order $N=1$ does not provide partitions since

Table 1. Partitions of the time points and Schrödinger perturbation terms for energy; orders $N$ from $N=1$ to $N=4 . S_{i}$ are the Huby-Tong numbers of the Schrödinger terms labeled by the index $i$; see (8). The abbreviation symbols are represented in (41)-(41c); see also [8] [9].

$$
\begin{aligned}
& N=1 \text { : no time points are present on the scale excepting for the beginning-end point; } S_{1}=1 \text {; } \\
& \text { Schrödinger perturbation energy: } \Delta E_{1}=\langle V\rangle \\
& N=2 \text { : only one time point is present on the scale beyond the beginning-end point; } S_{2}=1 \text {; } \\
& \text { Schrödinger perturbation energy: } \Delta E_{2}=\langle V P V\rangle \quad N=3, \quad N-1=2 ; \\
& \text { Partitions of } N-1 \\
& 11 \\
& 2 \\
& \text { Partitions of } N-1 \\
& 111 \\
& 21 \\
& 12 \\
& 3 \\
& S_{4}=S_{1}^{3}+S_{2} S_{1}+S_{1} S_{2}+S_{3}=1+1+1+2=5 \\
& \Delta E_{4}=\langle V P V P V P V\rangle-\Delta E_{1}\left\langle V P^{2} V P V\right\rangle-\Delta E_{1}\left\langle V P V P^{2} V\right\rangle-\Delta E_{2}\left\langle V P^{2} V\right\rangle+\left(\Delta E_{1}\right)^{2}\left\langle V P^{3} V\right\rangle \text {. }
\end{aligned}
$$


Table 2. Partitions of the time points and Schrödinger perturbation terms for energy; order $N=5 . S_{i}$ are the Huby-Tong numbers of the Schrödinger terms labeled by the index $i$; see (8). The abbreviation symbols are represented in (41)-(41c); see also [8] [9]. $N=5, \quad N-1=4$.

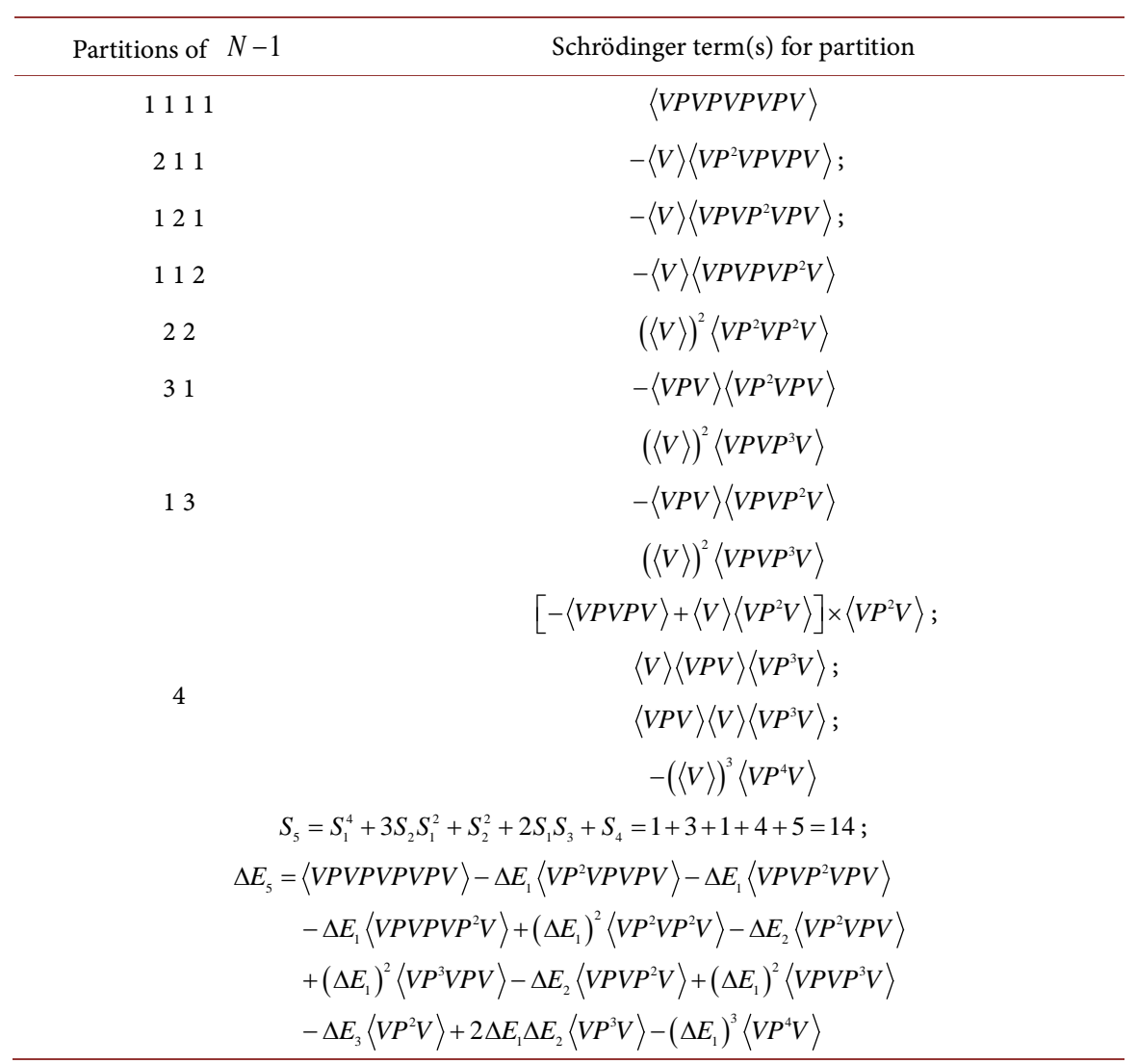

Table 3. Partitions of the time points and Schrödinger perturbation terms for energy; order $N=6 . S_{i}$ are the Huby-Tong numbers of the Schrödinger terms labeled by the index $i$; see (8). For the abbreviation symbols see (41)-(41c) and [8] [9].

\begin{tabular}{cc}
\hline Partitions of $N-1$ & Schrödinger term(s) for partition \\
\hline 11111 & $\langle V P V P V P V P V P V\rangle$ \\
2111 & $-\langle V\rangle\left\langle V P^{2} V P V P V P V\right\rangle$ \\
1211 & $-\langle V\rangle\left\langle V P V P^{2} V P V P V\right\rangle$ \\
1121 & $-\langle V\rangle\left\langle V P V P V P^{2} V P V\right\rangle$ \\
1112 & $-\langle V\rangle\left\langle V P V P V P V P^{2} V\right\rangle$ \\
221 & $(\langle V\rangle)^{2}\left\langle V P^{2} V P^{2} V P V\right\rangle$ \\
212 & $(\langle V\rangle)^{2}\left\langle V P^{2} V P V P^{2} V\right\rangle$ \\
122 & $(\langle V\rangle)^{2}\left\langle V P V P^{2} V P^{2} V\right\rangle$ \\
311 & $(\langle V\rangle)^{2}\left\langle V P^{3} V P V P V\right\rangle$ \\
& $-\langle V P V\rangle\left\langle V P^{2} V P V P V\right\rangle$ \\
131 & $(\langle V\rangle)^{2}\left\langle V P V P^{3} V P V\right\rangle$ \\
& $-\langle V P V\rangle\left\langle V P V P^{2} V P V\right\rangle$ \\
\hline
\end{tabular}




\section{Continued}

113

32

23

41

14

5 $(\langle V\rangle)^{2}\left\langle V P V P V P^{3} V\right\rangle$

$-\langle V P V\rangle\left\langle V P V P V P^{2} V\right\rangle$

$\langle V P V\rangle\langle V\rangle\left\langle V P^{2} V P^{2} V\right\rangle$

$-(\langle V\rangle)^{3}\left\langle V P^{3} V P^{2} V\right\rangle$

$\langle V P V\rangle\langle V\rangle\left\langle V P^{2} V P^{2} V\right\rangle$

$-(\langle V\rangle)^{3}\left\langle V P^{2} V P^{3} V\right\rangle$

$-\left\langle V P^{2} V P V\right\rangle\left(\langle V P V P V\rangle-\langle V\rangle\left\langle V P^{2} V\right\rangle\right)$

$\langle V\rangle\langle V P V\rangle\left\langle V P^{3} V P V\right\rangle$

$\langle V\rangle\langle V P V\rangle\left\langle V P^{3} V P V\right\rangle$

$-(\langle V\rangle)^{3}\left\langle V P^{4} V P V\right\rangle$

$-\left\langle V P^{2} V P V\right\rangle\left(\langle V P V P V\rangle-\langle V\rangle\left\langle V P^{2} V\right\rangle\right)$

$\langle V\rangle\langle V P V\rangle\left\langle V P V P^{3} V\right\rangle$

$\langle V\rangle\langle V P V\rangle\left\langle V P V P^{3} V\right\rangle$

$-(\langle V\rangle)^{3}\left\langle V P V P^{4} V\right\rangle$

$$
\begin{gathered}
-\Delta E_{4}\left\langle V P^{2} V\right\rangle \\
\Delta E_{1} \Delta E_{3}\left\langle V P^{3} V\right\rangle \\
\Delta E_{2} \Delta E_{2}\left\langle V P^{3} V\right\rangle \\
\Delta E_{3} \Delta E_{1}\left\langle V P^{3} V\right\rangle \\
-\left(\Delta E_{1}\right)^{2} \Delta E_{2}\left\langle V P^{4} V\right\rangle \\
-\left(\Delta E_{1}\right)^{2} \Delta E_{2}\left\langle V P^{4} V\right\rangle \\
-\left(\Delta E_{1}\right)^{2} \Delta E_{2}\left\langle V P^{4} V\right\rangle \\
\left(\Delta E_{1}\right)^{4}\left\langle V P^{5} V\right\rangle
\end{gathered}
$$$$
S_{5}=S_{4}+2 S_{1} S_{3}+S_{2}^{2}+3 S_{1}^{2} S_{2}+S_{1}^{4}=5+2 \times 2+1+3+1=14
$$$$
S_{6}=S_{1}^{5}+4 S_{1}^{3} S_{2}+3 S_{2}^{2} S_{1}+3 S_{3} S_{1}^{2}+2 S_{3} S_{2}+2 S_{4} S_{1}+S_{5}
$$$$
=1+4+3+3 \times 2+2 \times 2+2 \times 5+14=42
$$

$\Delta E_{6}=\langle V P V P V P V P V P V\rangle-\Delta E_{1}\left\langle V P^{2} V P V P V P V\right\rangle-\Delta E_{1}\left\langle V P V P^{2} V P V P V\right\rangle$

$-\Delta E_{1}\left\langle V P V P V P^{2} V P V\right\rangle-\Delta E_{1}\left\langle V P V P V P^{2} V\right\rangle+\left(\Delta E_{1}\right)^{2}\left\langle V P^{2} V P^{2} V P V\right\rangle$

$+\left(\Delta E_{1}\right)^{2}\left\langle V P^{2} V P V P^{2} V\right\rangle+\left(\Delta E_{1}\right)^{2}\left\langle V P V P^{2} V P^{2} V\right\rangle+\left(\Delta E_{1}\right)^{2}\left\langle V P^{3} V P V P V\right\rangle$

$-\Delta E_{2}\left\langle V P^{2} V P V P V\right\rangle+\left(\Delta E_{1}\right)^{2}\left\langle V P V P^{3} V P V\right\rangle-\Delta E_{2}\left\langle V P V P^{2} V P V\right\rangle$

$+\left(\Delta E_{1}\right)^{2}\left\langle V P V P V P^{3} V\right\rangle-\Delta E_{2}\left\langle V P V P V P^{2} V\right\rangle+\Delta E_{1} \Delta E_{2}\left\langle V P^{2} V P^{2} V\right\rangle$

$-\left(\Delta E_{1}\right)^{3}\left\langle V P^{3} V P^{2} V\right\rangle+\Delta E_{1} \Delta E_{2}\left\langle V P^{2} V P^{2} V\right\rangle-\left(\Delta E_{1}\right)^{3}\left\langle V P^{2} V P^{3} V\right\rangle$

$-\Delta E_{3}\left\langle V P^{2} V P V\right\rangle+2 \Delta E_{1} \Delta E_{2}\left\langle V P^{3} V P V\right\rangle-\left(\Delta E_{1}\right)^{3}\left\langle V P^{4} V P V\right\rangle$

$-\Delta E_{3}\left\langle V P V P^{2} V\right\rangle+2 \Delta E_{1} \Delta E_{2}\left\langle V P V P^{3} V\right\rangle-\left(\Delta E_{1}\right)^{3}\left\langle V P V P^{4} V\right\rangle$

$-\Delta E_{4}\left\langle V P^{2} V\right\rangle+2 \Delta E_{1} \Delta E_{3}\left\langle V P^{3} V\right\rangle+\left(\Delta E_{2}\right)^{2}\left\langle V P^{3} V\right\rangle$

$-3\left(\Delta E_{1}\right)^{2} \Delta E_{2}\left\langle V P^{4} V\right\rangle+\left(\Delta E_{1}\right)^{4}\left\langle V P^{5} V\right\rangle$ 
Table 4. Partitions of the time points and Schrödinger perturbation terms for energy; $N=7$. For the sake of brevity the individual Schrödinger terms are combined into energy terms. The Huby-Tong number of the Schrödinger perturbation terms $\left(S_{p}\right)$ is calculated separately for any partition.

\begin{tabular}{|c|c|c|}
\hline Partition & $S_{p}$ & Schrödinger energy term(s) \\
\hline 111111 & 1 & $\langle V P V P V P V P V P V P V\rangle$ \\
\hline 21111 & 1 & $-\Delta E_{1}\left\langle V P^{2} V P V P V P V P V\right\rangle$ \\
\hline 12111 & 1 & $-\Delta E_{1}\left\langle V P V P^{2} V P V P V P V\right\rangle$ \\
\hline 11211 & 1 & $-\Delta E_{1}\left\langle V P V P V P^{2} V P V P V\right\rangle$ \\
\hline 11121 & 1 & $-\Delta E_{1}\left\langle V P V P V P V P^{2} V P V\right\rangle$ \\
\hline 11112 & 1 & $-\Delta E_{1}\left\langle V P V P V P V P V P^{2} V\right\rangle$ \\
\hline 2211 & 1 & $\left(\Delta E_{1}\right)^{2}\left\langle V P^{2} V P^{2} V P V P V\right\rangle$ \\
\hline 2121 & 1 & $\left(\Delta E_{1}\right)^{2}\left\langle V P^{2} V P V P^{2} V P V\right\rangle$ \\
\hline 2112 & 1 & $\left(\Delta E_{1}\right)^{2}\left\langle V P^{2} V P V P V P^{2} V\right\rangle$ \\
\hline 1212 & 1 & $\left(\Delta E_{1}\right)^{2}\left\langle V P V P^{2} V P V P^{2} V\right\rangle$ \\
\hline 1122 & 1 & $\left(\Delta E_{1}\right)^{2}\left\langle V P V P V P^{2} V P^{2} V\right\rangle$ \\
\hline 1221 & 1 & $\left(\Delta E_{1}\right)^{2}\left\langle V P V P^{2} V P^{2} V P V\right\rangle$ \\
\hline \multirow[t]{2}{*}{3111} & 2 & $-\Delta E_{2}\left\langle V P^{2} V P V P V P V\right\rangle$ \\
\hline & & $+\left(\Delta E_{1}\right)^{2}\left\langle V P^{3} V P V P V P V\right\rangle$ \\
\hline \multirow[t]{2}{*}{1311} & 2 & $-\Delta E_{2}\left\langle V P V P^{2} V P V P V\right\rangle$ \\
\hline & & $+\left(\Delta E_{1}\right)^{2}\left\langle V P V P^{3} V P V P V\right\rangle$ \\
\hline \multirow[t]{2}{*}{1131} & 2 & $-\Delta E_{2}\left\langle V P V P V P^{2} V P V\right\rangle$ \\
\hline & & $+\left(\Delta E_{1}\right)^{2}\left\langle V P V P V P^{3} V P V\right\rangle$ \\
\hline \multirow[t]{2}{*}{1113} & 2 & $-\Delta E_{2}\left\langle V P V P V P V P^{2} V\right\rangle$ \\
\hline & & $+\left(\Delta E_{1}\right)^{2}\left\langle V P V P V P V P^{3} V\right\rangle$ \\
\hline \multirow[t]{2}{*}{321} & 2 & $\Delta E_{2} \Delta E_{1}\left\langle V P^{2} V P^{2} V P V\right\rangle$ \\
\hline & & $-\left(\Delta E_{1}\right)^{3}\left\langle V P^{3} V P^{2} V P V\right\rangle$ \\
\hline \multirow[t]{2}{*}{312} & 2 & $\Delta E_{2} \Delta E_{1}\left\langle V P^{2} V P V P^{2} V\right\rangle$ \\
\hline & & $-\left(\Delta E_{1}\right)^{3}\left\langle V P^{3} V P V P^{2} V\right\rangle$ \\
\hline \multirow[t]{2}{*}{231} & 2 & $\Delta E_{1} \Delta E_{2}\left\langle V P^{2} V P^{2} V P V\right\rangle$ \\
\hline & & $-\left(\Delta E_{1}\right)^{3}\left\langle V P^{2} V P^{3} V P V\right\rangle$ \\
\hline \multirow[t]{2}{*}{213} & 2 & $\Delta E_{1} \Delta E_{2}\left\langle V P^{2} V P V P^{2} V\right\rangle$ \\
\hline & & $-\left(\Delta E_{1}\right)^{3}\left\langle V P^{2} V P V P^{3} V\right\rangle$ \\
\hline \multirow[t]{2}{*}{132} & 2 & $\Delta E_{2} \Delta E_{1}\left\langle V P V P^{2} V P^{2} V\right\rangle$ \\
\hline & & $-\left(\Delta E_{1}\right)^{3}\left\langle V P V P^{3} V P^{2} V\right\rangle$ \\
\hline \multirow[t]{2}{*}{123} & 2 & $\Delta E_{1} \Delta E_{2}\left\langle V P V P^{2} V P^{2} V\right\rangle$ \\
\hline & & $-\left(\Delta E_{1}\right)^{3}\left\langle V P V P^{2} V P^{3} V\right\rangle$ \\
\hline
\end{tabular}




\section{Continued}

411

114

141

222

33

42

24

51

15

14
$-\Delta E_{3}\left\langle V P^{2} V P V P V\right\rangle$

$+\Delta E_{2} \Delta E_{1}\left\langle V P^{3} V P V P V\right\rangle$

$+\Delta E_{1} \Delta E_{2}\left\langle V P^{3} V P V P V\right\rangle$

$-\left(\Delta E_{1}\right)^{3}\left\langle V P^{4} V P V P V\right\rangle$

$-\Delta E_{3}\left\langle V P V P V P^{2} V\right\rangle$

$+\Delta E_{2} \Delta E_{1}\left\langle V P V P V P^{3} V\right\rangle$

$+\Delta E_{1} \Delta E_{2}\left\langle V P V P V P^{3} V\right\rangle$

$-\left(\Delta E_{1}\right)^{3}\left\langle V P V P V P^{4} V\right\rangle$

$-\Delta E_{3}\left\langle V P V P^{2} V P V\right\rangle$

$+\Delta E_{2} \Delta E_{1}\left\langle V P V P^{3} V P V\right\rangle$

$+\Delta E_{1} \Delta E_{2}\left\langle V P V P^{3} V P V\right\rangle$

$-\left(\Delta E_{1}\right)^{3}\left\langle V P V P^{4} V P V\right\rangle$

$-\left(\Delta E_{1}\right)^{3}\left\langle V P^{2} V P^{2} V P^{2} V\right\rangle$

$\Delta E_{2} \Delta E_{2}\left\langle V P^{2} V P^{2} V\right\rangle$

$-\left(\Delta E_{1}\right)^{2} \Delta E_{2}\left\langle V P^{3} V P^{2} V\right\rangle$

$-\Delta E_{2}\left(\Delta E_{1}\right)^{2}\left\langle V P^{2} V P^{3} V\right\rangle$

$\left(\Delta E_{1}\right)^{2}\left(\Delta E_{1}\right)^{2}\left\langle V P^{3} V P^{3} V\right\rangle$

$\Delta E_{3} \Delta E_{1}\left\langle V P^{2} V P^{2} V\right\rangle$

$-\left(\Delta E_{2}\right)^{2} \Delta E_{1}\left\langle V P^{3} V P^{2} V\right\rangle$

$-\left(\Delta E_{1}\right)^{2} \Delta E_{2}\left\langle V P^{3} V P^{2} V\right\rangle$

$\left(\Delta E_{1}\right)^{4}\left\langle V P^{4} V P^{2} V\right\rangle$

$\Delta E_{1} \Delta E_{3}\left\langle V P^{2} V P^{2} V\right\rangle$

$-\Delta E_{2}\left(\Delta E_{1}\right)^{2}\left\langle V P^{2} V P^{3} V\right\rangle$

$-\left(\Delta E_{1}\right)^{2} \Delta E_{2}\left\langle V P^{2} V P^{3} V\right\rangle$

$\left(\Delta E_{1}\right)^{4}\left\langle V P^{2} V P^{4} V\right\rangle$

$-\Delta E_{4}\left\langle V P^{2} V P V\right\rangle$

$\Delta E_{1} \Delta E_{3}\left\langle V P^{3} V P V\right\rangle$

$\Delta E_{3} \Delta E_{1}\left\langle V P^{3} V P V\right\rangle$

$\left(\Delta E_{2}\right)^{2}\left\langle V P^{3} V P V\right\rangle$

$-\left(\Delta E_{1}\right)^{2} \Delta E_{2}\left\langle V P^{4} V P V\right\rangle$

$-\Delta E_{1} \Delta E_{2} \Delta E_{1}\left\langle V P^{4} V P V\right\rangle$

$-\Delta E_{2}\left(\Delta E_{1}\right)^{2}\left\langle V P^{4} V P V\right\rangle$

$\left(\Delta E_{1}\right)^{4}\left\langle V P^{5} V P V\right\rangle$

$-\Delta E_{4}\left\langle V P V P^{2} V\right\rangle$

$\Delta E_{1} \Delta E_{3}\left\langle V P V P^{3} V\right\rangle$

$\Delta E_{3} \Delta E_{1}\left\langle V P V P^{3} V\right\rangle$

$\left(\Delta E_{2}\right)^{2}\left\langle V P V P^{3} V\right\rangle$ 


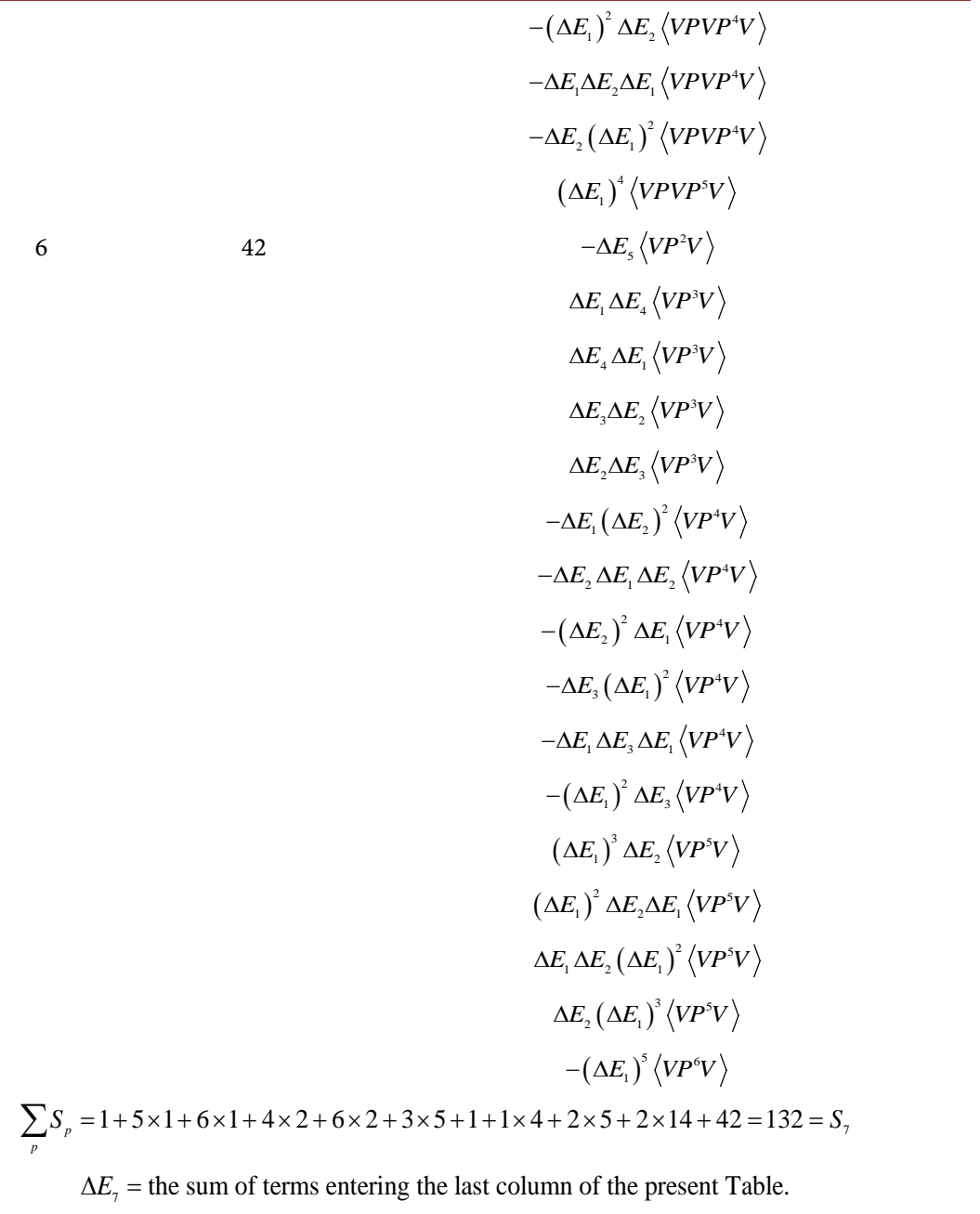

$N-1=0$ : the time loop has only one point on it which is the beginning-end point of the scale, and there exist no additional points of collisions with the perturbation potential. In Table 1 are presented partitions of $N-1$ for $N$ from 2 to 4 and the corresponding energy perturbation terms are given also there. In this case the abbreviations of energy expressions done in the former papers [8] [9] are applied. For example

$$
\langle V P V P V P V P V\rangle=\sum_{p} \sum_{q} \sum_{r} \sum_{s} \frac{U_{n p} U_{p q} U_{q r} U_{r s} U_{s n}}{\left(E_{n}-E_{p}\right)\left(E_{n}-E_{q}\right)\left(E_{n}-E_{r}\right)\left(E_{n}-E_{s}\right)}
$$

is the uncontracted perturbation term for $N=5$, where

$$
U_{a b}=\left\langle a\left|V^{\mathrm{per}}\right| b\right\rangle \text {. }
$$

An example of a contracted perturbation term representing a contribution of the main loop of time is

$$
\left\langle V P^{\alpha} V P^{\beta} V P^{\gamma} V \cdots\right\rangle
$$

where symbols $V$ replace the matrix elements of the kind given in (41a) and 


$$
P^{\alpha}=\frac{1}{\left(E_{n}-E_{p}\right)^{\alpha}}, \quad P^{\beta}=\frac{1}{\left(E_{n}-E_{q}\right)^{\beta}}, \quad P^{\gamma}=\frac{1}{\left(E_{n}-E_{r}\right)^{\gamma}}, \quad \cdots
$$

The summation symbols in (41) run over the unperturbed states being different than $n$. The data for $N$ from 5 to 7 are given in Tables 2-4.

The partition operations are of importance because they lead to the sets of time points which-when submitted to contractions-provide us readily with the Schrödinger perturbation terms. The corresponding rules are given in Section 7 . The summation symbols present in (41) indicate that the way along any main loop of time belonging to $N>1$ can be repeated in order to take into account more than one quantum state from the set of $p, q, r$, and $s$ states attainable, for example, in case of $N=5$.

In numerous cases the perturbation terms belonging to a given $N$ are found equal to products of the perturbation terms for energy having the order lower than $N$. Another simplification comes from the symmetry properties entering partitions: for large $N$ this gives a reduction of the number of terms necessary to calculate to about one half of the original number of the terms belonging to $N$.

\section{Contractions of the Time Points Entering the Sets Obtained Due to Partitions}

The contractions of the time points grouped in the sets due to partitions of $N-1$ in (40) can be best explained by an example. Let us consider partition of

$$
M=N-1
$$

into two sets, so

$$
M=Q+R
$$

therefore we have the time points labeled by

$$
1,2,3, \cdots, Q
$$

in the first set, and the time points labeled by

$$
1^{\prime}, 2^{\prime}, 3^{\prime}, \cdots, R
$$

in the second set. Evidently

$$
\begin{aligned}
& Q+1=1^{\prime}, \\
& Q+2=2^{\prime}, \\
& Q+3=3^{\prime},
\end{aligned}
$$

etc. Our aim is to examine the contractions of the time points entering set $Q$ and set $R$. To this purpose let us take for example

$$
Q=3, R=4 \text {. }
$$

This case corresponds to $N=8$ and is explicitly considered in Table 6 of the present paper.

The three time points of $Q$ can give contractions

$$
1: 3
$$


and

$$
1: 2: 3
$$

therefore the number of contractions is

$$
S_{Q}=S_{3}=2 .
$$

In a similar way the four points of set $R$ give contractions

$$
\begin{gathered}
1^{\prime}: 4^{\prime}, \\
1^{\prime}: 4^{\prime}-2^{\prime}: 3^{\prime}, \\
1^{\prime}: 2^{\prime}: 4^{\prime}, \\
1^{\prime}: 3^{\prime}: 4^{\prime}, \\
1^{\prime}: 2^{\prime}: 3^{\prime}: 4^{\prime},
\end{gathered}
$$

so together five contractions:

$$
S_{R}=S_{4}=5
$$

are present. Let us note that the end points 1 and 3 enter obligatorily contractions of set $Q$ and the end points $1^{\prime}$ and $4^{\prime}$ enter obligatorily contractions of set $R$. Because of

$$
S_{Q} \times S_{R}=2 \times 5=10,
$$

both sets $Q$ and $R$ give together 10 Schrödinger perturbation terms belonging to $N=8$ for the chosen example of $Q$ and $R$.

Moreover the absolute value of the Schrödinger perturbation term obtained in virtue of contraction (47) is equal to $\Delta E_{2}$ and a similar value due to contraction (47a) is equal to

$$
\Delta E_{1} \Delta E_{1}=\left(\Delta E_{1}\right)^{2} .
$$

Also contractions of the set $R$ give the energy terms. The two terms resulted from contractions (49) and (49a) can be combined into the energy term

$$
\Delta E_{3},
$$

the absolute value of the term given by contraction (49b) is equal to

$$
\Delta E_{1} \Delta E_{2},
$$

the absolute value of the term given by contraction (49c) is equal to

$$
\Delta E_{2} \Delta E_{1} \text {, }
$$

and the absolute value of the term given by contraction (49d) is equal to

$$
\Delta E_{1} \Delta E_{1} \Delta E_{1}=\left(\Delta E_{1}\right)^{3} .
$$

In general the contractions of sets belonging to $Q$ and $R$ provide us with the side loops of time. Such contractions give either the energy terms represented by these loops ( $N=1$ and $N=2$ ), or the Schrödinger perturbation terms which can be combined into the energy terms. Let us note that a maximal order of the perturbation energy given by the set $Q$ is

$$
Q-1=3-1=2 \text {, }
$$


and a maximal order of the perturbation energy given by set $R$ is

$$
R-1=4-1=3 \text {. }
$$

Any contribution given by the side loops should be supplemented by that given by the main loop of time. Single contractions like

$$
1: 3
$$

or

$$
1^{\prime}: 4^{\prime}
$$

give together the term

$$
\left\langle V P^{2} V P^{2} V\right\rangle
$$

double contractions like

$$
1: 2: 3
$$

combined with

$$
1^{\prime}: 2^{\prime}: 4^{\prime} \text { or } 1^{\prime}: 3^{\prime}: 4^{\prime}
$$

give the term

$$
\left\langle V P^{3} V P^{3} V\right\rangle
$$

A mixed order of contractions gives respectively mixed exponents of $P$ in the energy term given by the main loop of time. For example contractions of $1: 3$ and $1^{\prime}: 2^{\prime}: 3^{\prime}: 4^{\prime}$ give the energy contribution from the main loop of time equal to

$$
\left\langle V P^{2} V P^{4} V\right\rangle
$$

In general the mixed orders of contractions given by the sets of $Q$ and $R$ provide us with the terms

$$
\left\langle V P^{\alpha} V P^{\beta} V\right\rangle
$$

where

$$
\alpha, \beta=2
$$

for single contractions,

$$
\alpha, \beta=3
$$

for double contractions,

$$
\alpha, \beta=4
$$

for triple contractions, i.e. involving four time points, etc. The exponent of $P$ due to a combined contraction (49a) is such as for a single contraction, because only contraction $1^{\prime}: 4^{\prime}$ defines the side loop of time in this case.

The results obtained for two-component partitions (viz. $M=Q+R$ ) can be readily extended to many-component partitions of the number $M$.

The full perturbation terms are products of the terms obtained from the main loop of time and the side loops, respectively. The 10 terms obtained from the side loops in virtue of Equation (50) are in fact reduced to 8 terms because of the com- 
bination of two terms from the set $R$ into one term representing the perturbation energy $\Delta E_{3}$. Therefore the partition $(Q, R)=(3,4)$ gives the following Schrödinger perturbation terms belonging to the order $N=8$ :

$$
\left\langle V P^{2} V P^{2} V\right\rangle \Delta E_{2} \Delta E_{3}
$$

for contractions $1: 3$ and $1^{\prime}: 4^{\prime}$ combined with $1^{\prime}: 4^{\prime} \frown 2^{\prime}: 3^{\prime}$,

$$
-\left\langle V P^{2} V P^{3} V\right\rangle \Delta E_{2} \Delta E_{1} \Delta E_{2}
$$

for contractions $1: 3$ and $1^{\prime}: 2^{\prime}: 4^{\prime}$,

$$
-\left\langle V P^{2} V P^{3} V\right\rangle \Delta E_{2} \Delta E_{2} \Delta E_{1}
$$

for contractions $1: 3$ and $1^{\prime}: 3^{\prime}: 4^{\prime}$,

$$
\left\langle V P^{2} V P^{4} V\right\rangle \Delta E_{2}\left(\Delta E_{1}\right)^{3}
$$

for contractions $1: 3$ and $1^{\prime}: 2^{\prime}: 3^{\prime}: 4^{\prime}$,

$$
-\left\langle V P^{3} V P^{2} V\right\rangle\left(\Delta E_{1}\right)^{2} \Delta E_{3}
$$

for contractions $1: 2: 3$ and $1^{\prime}: 4^{\prime}$ combined with $1^{\prime}: 4^{\prime} \frown 2^{\prime}: 3^{\prime}$,

$$
\left\langle V P^{3} V P^{3} V\right\rangle\left(\Delta E_{1}\right)^{2} \Delta E_{1} \Delta E_{2}
$$

for contractions $1: 2: 3$ and $1^{\prime}: 2^{\prime}: 4^{\prime}$,

$$
\left\langle V P^{3} V P^{3} V\right\rangle\left(\Delta E_{1}\right)^{2} \Delta E_{2} \Delta E_{1}
$$

for contractions $1: 2: 3$ and $1^{\prime}: 3^{\prime}: 4^{\prime}$, and

$$
-\left\langle V P^{3} V P^{4} V\right\rangle\left(\Delta E_{1}\right)^{2}\left(\Delta E_{1}\right)^{3}
$$

for contractions $1: 2: 3$ and $1^{\prime}: 2^{\prime}: 3^{\prime}: 4^{\prime}$. Evidently some terms, like (71) and (72) or (75) and (76), become equal.

The sign of terms (70)-(77) is defined by the rule that the full time scale having an odd number of loops on its diagram gives a term with a plus sign, whereas for an even number of loops this scale gives the term with a minus sign.

\section{Partitions of $N-1$ Define the Terms Characteristic for the Perturbation Order $N$}

In our attempt to demonstrate that a suitable time scale can provide us with a correct series expansion of the Schrödinger perturbation terms for energy let us note that not all perturbation terms belonging to some $N$ are new: numerous of them-especially for large $N$-are combinations of terms obtained for the perturbation orders lower than $N$, i.e. they enter the terms belonging to $N^{\prime}<N$. The aim of the present Section is to select the terms which are specific for a given $N$ : they are such that no energy terms $\Delta E_{N^{\prime}}$, with $N^{\prime}<N$ excepting for a single

$$
\Delta E_{1} \text {, }
$$

or the powers of $\Delta E_{1}$ enter into them. 
The set of such specific, or characteristic terms, for a given $N$ can be obtained with the aid of the partition numbers of

$$
N-1 .
$$

The way to get them from the whole number $S_{N}$ of the terms belonging to the order $N$ is very simple: we choose from $S_{N}$ only the terms which are built up of $\Delta E_{N^{\prime}}=\Delta E_{1}$.

These terms-together with the basic perturbation term corresponding to the scale of $N$ for which no contraction points are considered-provide us with the characteristic terms for $N$. There exists a one-to-one correspondence of the characteristic terms for $N$ and partitions of $N-1$; see Table 5 .

The number of characteristic terms is equal to

$$
C_{N}=2^{N-2}
$$

and is evidently smaller than $S_{N}$. The ratios of $S_{N}$ and $C_{N}$ obtained for the neighbouring order numbers $N$ are respectively

$$
S_{N+1} / S_{N}=\frac{(2 N) !(N-1) !}{(2 N-2) !(N+1) !}=\frac{4-\frac{2}{N}}{1+\frac{1}{N}} \cong 4,
$$

and

$$
C_{N+1} / C_{N}=\frac{2^{N+1-2}}{2^{N-2}}=2 .
$$

Therefore $S_{N}$ grows up with $N$ more rapidly than $C_{N}$. Moreover-because of symmetry-the number of $C_{N}$ can be reduced to about one-half of its original value when $N$ is large.

\section{Large Partition Numbers and Their Contribution to the Schrödinger Perturbation Energy}

The order number $N$ means $N$ points of time present on the circular scale for that $N$ :

$$
1,2,3, \cdots, N \text {. }
$$

Let the $N$ th point be the beginning-end point on the scale, so this point is free from contractions. Then the largest partition number corresponding to $N$ is

$$
M=N-1 .
$$

For $N=7$ taken as an example this gives evidently $M=7-1=6$. The aim of the present Section is to present the contribution to the perturbation energy given by the partition number equal to $M$.

First let us note that contraction

$$
1: M=1:(N-1)
$$

gives the largest side loop corresponding to $N$. The number of the time points on this loop is

$$
M-1 .
$$


Table 5. Terms characteristic for the perturbation order $N$. They begin their existence in the perturbation series at a given $N$. The table presents the characteristic terms for $N=4,5$ and 6 . A common property of these terms is that they possess the multipliers being solely a power of $\langle V\rangle=\Delta E_{1}$. The number of characteristic terms is discussed in Section 8 .

\begin{tabular}{|c|c|c|}
\hline \multirow[t]{5}{*}{$N=4$} & Partition of $N-1$ & Characteristic term \\
\hline & 111 & $\langle V P V P V P V\rangle$ \\
\hline & 21 & $-\Delta E_{1}\left\langle V P^{2} V P V\right\rangle$ \\
\hline & 12 & $-\Delta E_{1}\left\langle V P V P^{2} V\right\rangle$ \\
\hline & 3 & $\left(\Delta E_{1}\right)^{2}\left\langle V P^{3} V\right\rangle$ \\
\hline \multirow{9}{*}{$N=5$} & Partition of $N-1$ & Characteristic term \\
\hline & 1111 & $\langle V P V P V P V P V\rangle$ \\
\hline & 211 & $-\Delta E_{1}\left\langle V P^{2} V P V P V\right\rangle$ \\
\hline & 121 & $-\Delta E_{1}\left\langle V P V P^{2} V P V\right\rangle$ \\
\hline & 112 & $-\Delta E_{1}\left\langle V P V P V P^{2} V\right\rangle$ \\
\hline & 22 & $\left(\Delta E_{1}\right)^{2}\left\langle V P^{2} V P^{2} V\right\rangle$ \\
\hline & 31 & $\left(\Delta E_{1}\right)^{2}\left\langle V P^{3} V P^{1} V\right\rangle$ \\
\hline & 13 & $\left(\Delta E_{1}\right)^{2}\left\langle V P V P^{3} V\right\rangle$ \\
\hline & 4 & $-\left(\Delta E_{1}\right)^{3}\left\langle V P^{4} V\right\rangle$ \\
\hline \multirow[t]{17}{*}{$N=6$} & Partition of $N-1$ & Characteristic term \\
\hline & 11111 & $\langle V P V P V P V P V P V\rangle$ \\
\hline & 2111 & $-\Delta E_{1}\left\langle V P^{2} V P V P V P V\right\rangle$ \\
\hline & 1211 & $-\Delta E_{1}\left\langle V P V P^{2} V P V P V\right\rangle$ \\
\hline & 1121 & $-\Delta E_{1}\left\langle V P V P V P^{2} V P V\right\rangle$ \\
\hline & 1112 & $-\Delta E_{1}\left\langle V P V P V P V P^{2} V\right\rangle$ \\
\hline & 221 & $\left(\Delta E_{1}\right)^{2}\left\langle V P^{2} V P^{2} V P V\right\rangle$ \\
\hline & 212 & $\left(\Delta E_{1}\right)^{2}\left\langle V P^{2} V P V P^{2} V\right\rangle$ \\
\hline & 122 & $\left(\Delta E_{1}\right)^{2}\left\langle V P V P^{2} V P^{2} V\right\rangle$ \\
\hline & 311 & $\left(\Delta E_{1}\right)^{2}\left\langle V P^{3} V P V P V\right\rangle$ \\
\hline & 131 & $\left(\Delta E_{1}\right)^{2}\left\langle V P V P^{3} V P V\right\rangle$ \\
\hline & 113 & $\left(\Delta E_{1}\right)^{2}\left\langle V P V P V P^{3} V\right\rangle$ \\
\hline & 32 & $-\left(\Delta E_{1}\right)^{3}\left\langle V P^{3} V P^{2} V\right\rangle$ \\
\hline & 23 & $-\left(\Delta E_{1}\right)^{3}\left\langle V P^{2} V P^{3} V\right\rangle$ \\
\hline & 41 & $-\left(\Delta E_{1}\right)^{3}\left\langle V P^{4} V P V\right\rangle$ \\
\hline & 14 & $-\left(\Delta E_{1}\right)^{3}\left\langle V P V P^{4} V\right\rangle$ \\
\hline & 5 & $\left(\Delta E_{1}\right)^{4}\left\langle V P^{5} V\right\rangle$ \\
\hline
\end{tabular}

If, in their turn, no supplementary contractions are taken into account, the side loop given by contraction (85) represents the uncontracted loop of the scale 
corresponding to the perturbation order of energy equal to

$$
M-1=N-2 .
$$

In effect the uncontracted side loop due to contraction (85) provides us with the first contribution to the perturbation energy equal to

$$
-\Delta E_{M-1}\left\langle V P^{2} V\right\rangle \text {. }
$$

The next contributions to the perturbation energy due to $M$ are given by triple contractions which are

$$
\begin{aligned}
& 1: 2: M, \\
& 1: 3: M, \\
& 1: 4: M, \\
& \quad \vdots \\
& 1: M-1: M .
\end{aligned}
$$

Such contractions will provide us with the energy terms

$$
\begin{aligned}
& \Delta E_{1} \Delta E_{M-2}\left\langle V P^{3} V\right\rangle, \\
& \Delta E_{2} \Delta E_{M-3}\left\langle V P^{3} V\right\rangle, \\
& \Delta E_{3} \Delta E_{M-4}\left\langle V P^{3} V\right\rangle,
\end{aligned}
$$

etc., on one side, and symmetrical terms to (90), viz.

$$
\begin{aligned}
& \Delta E_{M-4} \Delta E_{3}\left\langle V P^{3} V\right\rangle, \\
& \Delta E_{M-3} \Delta E_{2}\left\langle V P^{3} V\right\rangle, \\
& \Delta E_{M-2} \Delta E_{1}\left\langle V P^{3} V\right\rangle,
\end{aligned}
$$

on the other side. The contraction process of (89) should be prolongated by other contractions than triple ones to a situation when the last component of energy due to $M$ is attained: this component is of the form

$$
\pm\left(\Delta E_{1}\right)^{M-1}\left\langle V P^{M} V\right\rangle
$$

see Table 7. A full number of the perturbation terms entering Table 7 is equal to $S_{N-1}=S_{6}=42$.

An important point of calculations is that the energy terms presented on the left-hand side of the first column given in Table 7 are characteristic for partition number 6 independently of the position which this 6 attains in the partition process of $N-1$. For example for $N=8$, so here $M=7$, the partition number 6 is entering the following partitions of $M$ :

$$
6 \quad 1
$$

and

16.

The energy terms entering the left side of the main column in Table 6 will be 
Table 6. Partitions of the time-points number and the Schrödinger perturbation terms for energy; $N=8$. For the sake of brevity the individual Schrödinger terms are combined into energy terms. The Huby-Tong number of the Schrödinger perturbation terms $\left(S_{p}\right)$ is calculated for any partition as a product of $S_{i}$ given by numbers $i$ entering the partitions of $N-1=7$.

\begin{tabular}{|c|c|c|}
\hline Partition & $S_{p}$ & Schrödinger energy term $(\mathrm{s})$ \\
\hline 1111111 & 1 & $\langle V P V P V P V P V P V P V\rangle$ \\
\hline 211111 & 1 & $-\Delta E_{1}\left\langle V P^{2} V P V P V P V P V P V\right\rangle$ \\
\hline 121111 & 1 & $-\Delta E_{1}\left\langle V P V P^{2} V P V P V P V P V\right\rangle$ \\
\hline 112111 & 1 & $-\Delta E_{1}\left\langle V P V P V P^{2} V P V P V P V\right\rangle$ \\
\hline 111211 & 1 & $-\Delta E_{1}\left\langle V P V P V P V P^{2} V P V P V\right\rangle$ \\
\hline 111121 & 1 & $-\Delta E_{1}\left\langle V P V P V P V P V P^{2} V P V\right\rangle$ \\
\hline 111112 & 1 & $-\Delta E_{1}\left\langle V P V P V P V P V P V P^{2} V\right\rangle$ \\
\hline 22111 & 1 & $\left(\Delta E_{1}\right)^{2}\left\langle V P^{2} V P^{2} V P V P V P V\right\rangle$ \\
\hline 12211 & 1 & $\left(\Delta E_{1}\right)^{2}\left\langle V P V P^{2} V P^{2} V P V P V\right\rangle$ \\
\hline 11221 & 1 & $\left(\Delta E_{1}\right)^{2}\left\langle V P V P V P^{2} V P^{2} V P V\right\rangle$ \\
\hline 11122 & 1 & $\left(\Delta E_{1}\right)^{2}\left\langle V P V P V P V P^{2} V P^{2} V\right\rangle$ \\
\hline 21211 & 1 & $\left(\Delta E_{1}\right)^{2}\left\langle V P^{2} V P V P^{2} V P V P V\right\rangle$ \\
\hline 21121 & 1 & $\left(\Delta E_{1}\right)^{2}\left\langle V P^{2} V P V P V P^{2} V P V\right\rangle$ \\
\hline 12112 & 1 & $\left(\Delta E_{1}\right)^{2}\left\langle V P V P^{2} V P V P V P^{2} V\right\rangle$ \\
\hline 21112 & 1 & $\left(\Delta E_{1}\right)^{2}\left\langle V P^{2} V P V P V P V P^{2} V\right\rangle$ \\
\hline 12121 & 1 & $\left(\Delta E_{1}\right)^{2}\left\langle V P V P^{2} V P V P^{2} V P V\right\rangle$ \\
\hline 11212 & 1 & $\left(\Delta E_{1}\right)^{2}\left\langle V P V P V P^{2} V P V P^{2} V\right\rangle$ \\
\hline 2221 & 1 & $-\left(\Delta E_{1}\right)^{3}\left\langle V P^{2} V P^{2} V P^{2} V P V\right\rangle$ \\
\hline 2212 & 1 & $-\left(\Delta E_{1}\right)^{3}\left\langle V P^{2} V P^{2} V P V P^{2} V\right\rangle$ \\
\hline 2122 & 1 & $-\left(\Delta E_{1}\right)^{3}\left\langle V P^{2} V P V P^{2} V P^{2} V\right\rangle$ \\
\hline 1222 & 1 & $-\left(\Delta E_{1}\right)^{3}\left\langle V P V P^{2} V P^{2} V P^{2} V\right\rangle$ \\
\hline \multirow[t]{2}{*}{3211} & 2 & $\Delta E_{2} \Delta E_{1}\left\langle V P^{2} V P^{2} V P V P V\right\rangle$ \\
\hline & & $-\left(\Delta E_{1}\right)^{3}\left\langle V P^{3} V P^{2} V P V P V\right\rangle$ \\
\hline \multirow[t]{2}{*}{3121} & 2 & $\Delta E_{2} \Delta E_{1}\left\langle V P^{2} V P V P^{2} V P V\right\rangle$ \\
\hline & & $-\left(\Delta E_{1}\right)^{3}\left\langle V P^{3} V P V P^{2} V P V\right\rangle$ \\
\hline \multirow[t]{2}{*}{3112} & 2 & $\Delta E_{2} \Delta E_{1}\left\langle V P^{2} V P V P V P^{2} V\right\rangle$ \\
\hline & & $-\left(\Delta E_{1}\right)^{3}\left\langle V P^{3} V P V P V P^{2} V\right\rangle$ \\
\hline \multirow[t]{2}{*}{2311} & 2 & $\Delta E_{1} \Delta E_{2}\left\langle V P^{2} V P^{2} V P V P V\right\rangle$ \\
\hline & & $-\left(\Delta E_{1}\right)^{3}\left\langle V P^{2} V P^{3} V P V P V\right\rangle$ \\
\hline \multirow[t]{2}{*}{1321} & 2 & $\Delta E_{2} \Delta E_{1}\left\langle V P V P^{2} V P^{2} V P V\right\rangle$ \\
\hline & & $-\left(\Delta E_{1}\right)^{3}\left\langle V P V P^{3} V P^{2} V P V\right\rangle$ \\
\hline \multirow[t]{2}{*}{1312} & 2 & $\Delta E_{2} \Delta E_{1}\left\langle V P V P^{2} V P V P^{2} V\right\rangle$ \\
\hline & & $-\left(\Delta E_{1}\right)^{3}\left\langle V P V P^{3} V P V P^{2} V\right\rangle$ \\
\hline
\end{tabular}




\begin{tabular}{|c|c|c|}
\hline Continued & & \\
\hline 2131 & 2 & $\Delta E_{1} \Delta E_{2}\left\langle V P^{2} V P V P^{2} V P V\right\rangle$ \\
\hline & & $-\left(\Delta E_{1}\right)^{3}\left\langle V P^{2} V P V P^{3} V P V\right\rangle$ \\
\hline 1231 & 2 & $\Delta E_{1} \Delta E_{2}\left\langle V P V P^{2} V P^{2} V P V\right\rangle$ \\
\hline & & $-\left(\Delta E_{1}\right)^{3}\left\langle V P V P^{2} V P^{3} V P V\right\rangle$ \\
\hline 1132 & 2 & $\Delta E_{2} \Delta E_{1}\left\langle V P V P V P^{2} V P^{2} V\right\rangle$ \\
\hline & & $-\left(\Delta E_{1}\right)^{3}\left\langle V P V P V P^{3} V P^{2} V\right\rangle$ \\
\hline 2113 & 2 & $\Delta E_{1} \Delta E_{2}\left\langle V P^{2} V P V P V P^{2} V\right\rangle$ \\
\hline & & $-\left(\Delta E_{1}\right)^{3}\left\langle V P^{2} V P V P V P^{3} V\right\rangle$ \\
\hline 1213 & 2 & $\Delta E_{1} \Delta E_{2}\left\langle V P V P^{2} V P V P^{2} V\right\rangle$ \\
\hline & & $-\left(\Delta E_{1}\right)^{3}\left\langle V P V P^{2} V P V P^{3} V\right\rangle$ \\
\hline 1123 & 2 & $\Delta E_{1} \Delta E_{2}\left\langle V P V P V P^{2} V P^{2} V\right\rangle$ \\
\hline & & $-\left(\Delta E_{1}\right)^{3}\left\langle V P V P V P^{2} V P^{3} V\right\rangle$ \\
\hline 31111 & 2 & $-\Delta E_{2}\left\langle V P^{2} V P V P V P V P V\right\rangle$ \\
\hline & & $\left(\Delta E_{1}\right)^{2}\left\langle V P^{3} V P V P V P V P V\right\rangle$ \\
\hline 13111 & 2 & $-\Delta E_{2}\left\langle V P V P^{2} V P V P V P V\right\rangle$ \\
\hline & & $\left(\Delta E_{1}\right)^{2}\left\langle V P V P^{3} V P V P V P V\right\rangle$ \\
\hline 11311 & 2 & $-\Delta E_{2}\left\langle V P V P V P^{2} V P V P V\right\rangle$ \\
\hline & & $\left(\Delta E_{1}\right)^{2}\left\langle V P V P V P^{3} V P V P V\right\rangle$ \\
\hline 11131 & 2 & $-\Delta E_{2}\left\langle V P V P V P V P^{2} V P V\right\rangle$ \\
\hline & & $\left(\Delta E_{1}\right)^{2}\left\langle V P V P V P V P^{3} V P V\right\rangle$ \\
\hline 11113 & 2 & $-\Delta E_{2}\left\langle V P V P V P V P V P^{2} V\right\rangle$ \\
\hline & & $\left(\Delta E_{1}\right)^{2}\left\langle V P V P V P V P V P^{3} V\right\rangle$ \\
\hline 223 & 2 & $-\left(\Delta E_{1}\right)^{2} \Delta E_{2}\left\langle V P^{2} V P^{2} V P^{2} V\right\rangle$ \\
\hline & & $\left(\Delta E_{1}\right)^{4}\left\langle V P^{2} V P^{2} V P^{3} V\right\rangle$ \\
\hline 232 & 2 & $-\left(\Delta E_{1}\right)^{2} \Delta E_{2}\left\langle V P^{2} V P^{2} V P^{2} V\right\rangle$ \\
\hline & & $\left(\Delta E_{1}\right)^{4}\left\langle V P^{2} V P^{3} V P^{2} V\right\rangle$ \\
\hline 322 & 2 & $-\left(\Delta E_{1}\right)^{2} \Delta E_{2}\left\langle V P^{2} V P^{2} V P^{2} V\right\rangle$ \\
\hline & & $\left(\Delta E_{1}\right)^{4}\left\langle V P^{3} V P^{2} V P^{2} V\right\rangle$ \\
\hline 331 & 4 & $\left(\Delta E_{2}\right)^{2}\left\langle V P^{2} V P^{2} V P V\right\rangle$ \\
\hline & & $-\Delta E_{2}\left(\Delta E_{1}\right)^{2}\left\langle V P^{2} V P^{3} V P V\right\rangle$ \\
\hline & & $-\left(\Delta E_{1}\right)^{2} \Delta E_{2}\left\langle V P^{3} V P^{2} V P V\right\rangle$ \\
\hline & & $\left(\Delta E_{1}\right)^{4}\left\langle V P^{3} V P^{3} V P V\right\rangle$ \\
\hline 313 & 4 & $\left(\Delta E_{2}\right)^{2}\left\langle V P^{2} V P V P^{2} V\right\rangle$ \\
\hline & & $-\Delta E_{2}\left(\Delta E_{1}\right)^{2}\left\langle V P^{2} V P V P^{3} V\right\rangle$ \\
\hline & & $-\left(\Delta E_{1}\right)^{2} \Delta E_{2}\left\langle V P^{3} V P V P^{2} V\right\rangle$ \\
\hline & & $\left(\Delta E_{1}\right)^{4}\left\langle V P^{3} V P V P^{3} V\right\rangle$ \\
\hline 133 & 4 & $\left(\Delta E_{2}\right)^{2}\left\langle V P V P^{2} V P^{2} V\right\rangle$ \\
\hline
\end{tabular}




\section{Continued}

4111

1411

1141

1114

421

412

241

142

214
5

5

5

5

5
$-\Delta E_{2}\left(\Delta E_{1}\right)^{2}\left\langle V P V P^{2} V P^{3} V\right\rangle$

$-\left(\Delta E_{1}\right)^{2} \Delta E_{2}\left\langle V P V P^{3} V P^{2} V\right\rangle$

$\left(\Delta E_{1}\right)^{4}\left\langle V P V P^{3} V P^{3} V\right\rangle$

$-\Delta E_{3}\left\langle V P^{2} V P V P V P V\right\rangle$

$\Delta E_{1} \Delta E_{2}\left\langle V P^{3} V P V P V P V\right\rangle$

$\Delta E_{2} \Delta E_{1}\left\langle V P^{3} V P V P V P V\right\rangle$

$-\left(\Delta E_{1}\right)^{3}\left\langle V P^{4} V P V P V P V\right\rangle$

$-\triangle E_{3}\left\langle V P V P^{2} V P V P V\right\rangle$

$\Delta E_{1} \Delta E_{2}\left\langle V P V P^{3} V P V P V\right\rangle$

$\Delta E_{2} \Delta E_{1}\left\langle V P V P^{3} V P V P V\right\rangle$

$-\left(\Delta E_{1}\right)^{3}\left\langle V P V P^{4} V P V P V\right\rangle$

$-\triangle E_{3}\left\langle V P V P V P^{2} V P V\right\rangle$

$\Delta E_{1} \Delta E_{2}\left\langle V P V P V P^{3} V P V\right\rangle$

$\Delta E_{2} \Delta E_{1}\left\langle V P V P V P^{3} V P V\right\rangle$

$-\left(\Delta E_{1}\right)^{3}\left\langle V P V P V P^{4} V P V\right\rangle$

$-\triangle E_{3}\left\langle V P V P V P V P^{2} V\right\rangle$

$\Delta E_{1} \Delta E_{2}\left\langle V P V P V P V P^{3} V\right\rangle$

$\Delta E_{2} \Delta E_{1}\left\langle V P V P V P V P^{3} V\right\rangle$

$-\left(\Delta E_{1}\right)^{3}\left\langle V P V P V P V P^{4} V\right\rangle$

$\Delta E_{3} \Delta E_{1}\left\langle V P^{2} V P^{2} V P V\right\rangle$

$-\left(\Delta E_{1}\right)^{3} \Delta E_{2}\left\langle V P^{3} V P^{2} V P V\right\rangle$

$-\Delta E_{2}\left(\Delta E_{1}\right)^{2}\left\langle V P^{3} V P^{2} V P V\right\rangle$

$\left(\Delta E_{1}\right)^{4}\left\langle V P^{4} V P^{2} V P V\right\rangle$

$\Delta E_{3} \Delta E_{1}\left\langle V P^{2} V P V P^{2} V\right\rangle$

$-\left(\Delta E_{1}\right)^{2} \Delta E_{2}\left\langle V P^{3} V P V P^{2} V\right\rangle$

$-\Delta E_{2}\left(\Delta E_{1}\right)^{2}\left\langle V P^{3} V P V P^{2} V\right\rangle$

$\left(\Delta E_{1}\right)^{4}\left\langle V P^{4} V P V P^{2} V\right\rangle$

$\Delta E_{1} \Delta E_{3}\left\langle V P^{2} V P^{2} V P V\right\rangle$

$-\left(\Delta E_{1}\right)^{2} \Delta E_{2}\left\langle V P^{2} V P^{3} V P V\right\rangle$

$-\Delta E_{2}\left(\Delta E_{1}\right)^{2}\left\langle V P^{2} V P^{3} V P V\right\rangle$

$\left(\Delta E_{1}\right)^{4}\left\langle V P^{2} V P^{4} V P V\right\rangle$

$\Delta E_{1} \Delta E_{3}\left\langle V P V P^{2} V P^{2} V\right\rangle$

$-\left(\Delta E_{1}\right)^{2} \Delta E_{2}\left\langle V P V P^{3} V P^{2} V\right\rangle$

$-\Delta E_{2}\left(\Delta E_{1}\right)^{2}\left\langle V P V P^{3} V P^{2} V\right\rangle$

$\left(\Delta E_{1}\right)^{4}\left\langle V P V P^{4} V P^{2} V\right\rangle$

$\Delta E_{1} \Delta E_{3}\left\langle V P^{2} V P V P^{2} V\right\rangle$

$-\left(\Delta E_{1}\right)^{2} \Delta E_{2}\left\langle V P^{2} V P V P^{3} V\right\rangle$ 


\section{Continued}

124

511

151

115

43

10
$-\Delta E_{2}\left(\Delta E_{1}\right)^{2}\left\langle V P^{2} V P V P^{3} V\right\rangle$

$\left(\Delta E_{1}\right)^{4}\left\langle V P^{2} V P V P^{4} V\right\rangle$

$\Delta E_{1} \Delta E_{3}\left\langle V P V P^{2} V P^{2} V\right\rangle$

$-\left(\Delta E_{1}\right)^{2} \Delta E_{2}\left\langle V P V P^{2} V P^{3} V\right\rangle$

$-\Delta E_{2}\left(\Delta E_{1}\right)^{2}\left\langle V P V P^{2} V P^{3} V\right\rangle$

$\left(\Delta E_{1}\right)^{4}\left\langle V P V P^{2} V P^{4} V\right\rangle$

$-\Delta E_{4}\left\langle V P^{2} V P V P V\right\rangle$

$\Delta E_{1} \Delta E_{3}\left\langle V P^{3} V P V P V\right\rangle$

$\left(\Delta E_{2}\right)^{2}\left\langle V P^{3} V P V P V\right\rangle$

$\Delta E_{3} \Delta E_{1}\left\langle V P^{3} V P V P V\right\rangle$

$-\left(\Delta E_{1}\right)^{2} \Delta E_{2}\left\langle V P^{4} V P V P V\right\rangle$

$-\Delta E_{1} \Delta E_{2} \Delta E_{1}\left\langle V P^{4} V P V P V\right\rangle$

$-\Delta E_{2}\left(\Delta E_{1}\right)^{2}\left\langle V P^{4} V P V P V\right\rangle$

$\left(\Delta E_{1}\right)^{4}\left\langle V P^{5} V P V P V\right\rangle$

$-\Delta E_{4}\left\langle V P V P^{2} V P V\right\rangle$

$\Delta E_{1} \Delta E_{3}\left\langle V P V P^{3} V P V\right\rangle$

$\left(\Delta E_{2}\right)^{2}\left\langle V P V P^{3} V P V\right\rangle$

$\Delta E_{3} \Delta E_{1}\left\langle V P V P^{3} V P V\right\rangle$

$-\left(\Delta E_{1}\right)^{2} \Delta E_{2}\left\langle V P V P^{4} V P V\right\rangle$

$-\Delta E_{1} \Delta E_{2} \Delta E_{1}\left\langle V P V P^{4} V P V\right\rangle$

$-\Delta E_{2}\left(\Delta E_{1}\right)^{2}\left\langle V P V P^{4} V P V\right\rangle$

$\left(\Delta E_{1}\right)^{4}\left\langle V P V P^{5} V P V\right\rangle$

$-\Delta E_{4}\left\langle V P V P V P^{2} V\right\rangle$

$\Delta E_{1} \Delta E_{3}\left\langle V P V P V P^{3} V\right\rangle$

$\left(\Delta E_{2}\right)^{2}\left\langle V P V P V P^{3} V\right\rangle$

$\Delta E_{3} \Delta E_{1}\left\langle V P V P V P^{3} V\right\rangle$

$-\left(\Delta E_{1}\right)^{2} \Delta E_{2}\left\langle V P V P V P^{4} V\right\rangle$

$-\Delta E_{1} \Delta E_{2} \Delta E_{1}\left\langle V P V P V P^{4} V\right\rangle$

$-\Delta E_{2}\left(\Delta E_{1}\right)^{2}\left\langle V P V P V P^{4} V\right\rangle$

$\left(\Delta E_{1}\right)^{4}\left\langle V P V P V P^{5} V\right\rangle$

$\Delta E_{3} \Delta E_{2}\left\langle V P^{2} V P^{2} V\right\rangle$

$-\Delta E_{3}\left(\Delta E_{1}\right)^{2}\left\langle V P^{2} V P^{3} V\right\rangle$

$-\Delta E_{2} \Delta E_{1} \Delta E_{2}\left\langle V P^{3} V P^{2} V\right\rangle$

$\Delta E_{2} \Delta E_{1}\left(\Delta E_{1}\right)^{2}\left\langle V P^{3} V P^{3} V\right\rangle$

$-\Delta E_{1}\left(\Delta E_{2}\right)^{2}\left\langle V P^{3} V P^{2} V\right\rangle$

$\Delta E_{1} \Delta E_{2}\left(\Delta E_{1}\right)^{2}\left\langle V P^{3} V P^{3} V\right\rangle$

$\left(\Delta E_{1}\right)^{3} \Delta E_{2}\left\langle V P^{4} V P^{2} V\right\rangle$ 


\section{Continued}

52

25

61

14

5

5

2

2

1

1

1

2

2

2

1
$-\left(\Delta E_{1}\right)^{5}\left\langle V P^{4} V P^{3} V\right\rangle$

$\Delta E_{2} \Delta E_{3}\left\langle V P^{2} V P^{2} V\right\rangle$

$-\left(\Delta E_{1}\right)^{2} \Delta E_{3}\left\langle V P^{3} V P^{2} V\right\rangle$

$-\Delta E_{2} \Delta E_{1} \Delta E_{2}\left\langle V P^{2} V P^{3} V\right\rangle$

$\left(\Delta E_{1}\right)^{3} \Delta E_{2}\left\langle V P^{2} V P^{4} V\right\rangle$

$-\left(\Delta E_{2}\right)^{2} \Delta E_{1}\left\langle V P^{2} V P^{3} V\right\rangle$

$\left(\Delta E_{1}\right)^{2} \Delta E_{2} \Delta E_{1}\left\langle V P^{3} V P^{3} V\right\rangle$

$\Delta E_{2}\left(\Delta E_{1}\right)^{3}\left\langle V P^{3} V P^{3} V\right\rangle$

$-\left(\Delta E_{1}\right)^{5}\left\langle V P^{3} V P^{4} V\right\rangle$

$\Delta E_{4} \Delta E_{1}\left\langle V P^{2} V P^{2} V\right\rangle$

$-\left(\Delta E_{1}\right)^{2} \Delta E_{3}\left\langle V P^{3} V P^{2} V\right\rangle$

$-\left(\Delta E_{2}\right)^{2} \Delta E_{1}\left\langle V P^{3} V P^{2} V\right\rangle$

$-\left(\Delta E_{1}\right)^{2} \Delta E_{3}\left\langle V P^{3} V P^{2} V\right\rangle$

$\left(\Delta E_{1}\right)^{3} \Delta E_{2}\left\langle V P^{4} V P^{2} V\right\rangle$

$\left(\Delta E_{1}\right)^{3} \Delta E_{2}\left\langle V P^{4} V P^{2} V\right\rangle$

$\left(\Delta E_{1}\right)^{3} \Delta E_{2}\left\langle V P^{4} V P^{2} V\right\rangle$

$-\left(\Delta E_{1}\right)^{5}\left\langle V P^{5} V P^{2} V\right\rangle$

$\Delta E_{1} \Delta E_{4}\left\langle V P^{2} V P^{2} V\right\rangle$

$-\Delta E_{3}\left(\Delta E_{1}\right)^{2}\left\langle V P^{2} V P^{3} V\right\rangle$

$-\Delta E_{1}\left(\Delta E_{2}\right)^{2}\left\langle V P^{2} V P^{3} V\right\rangle$

$-\Delta E_{3}\left(\Delta E_{1}\right)^{2}\left\langle V P^{2} V P^{3} V\right\rangle$

$\Delta E_{2}\left(\Delta E_{1}\right)^{3}\left\langle V P^{2} V P^{4} V\right\rangle$

$\Delta E_{2}\left(\Delta E_{1}\right)^{3}\left\langle V P^{2} V P^{4} V\right\rangle$

$\Delta E_{2}\left(\Delta E_{1}\right)^{3}\left\langle V P^{2} V P^{4} V\right\rangle$

$-\left(\Delta E_{1}\right)^{5}\left\langle V P^{2} V P^{5} V\right\rangle$

$-\Delta E_{5}\left\langle V P^{2} V P V\right\rangle$

$\Delta E_{1} \Delta E_{4}\left\langle V P^{3} V P V\right\rangle$

$\Delta E_{4} \Delta E_{1}\left\langle V P^{3} V P V\right\rangle$

$\Delta E_{3} \Delta E_{2}\left\langle V P^{3} V P V\right\rangle$

$\Delta E_{2} \Delta E_{3}\left\langle V P^{3} V P V\right\rangle$

$-\Delta E_{1}\left(\Delta E_{2}\right)^{2}\left\langle V P^{4} V P V\right\rangle$

$-\Delta E_{2} \Delta E_{1} \Delta E_{2}\left\langle V P^{4} V P V\right\rangle$

$-\left(\Delta E_{2}\right)^{2} \Delta E_{1}\left\langle V P^{4} V P V\right\rangle$

$-\Delta E_{3}\left(\Delta E_{1}\right)^{2}\left\langle V P^{4} V P V\right\rangle$

$-\Delta E_{1} \Delta E_{3} \Delta E_{1}\left\langle V P^{4} V P V\right\rangle$

$-\left(\Delta E_{1}\right)^{2} \Delta E_{3}\left\langle V P^{4} V P V\right\rangle$

$\left(\Delta E_{1}\right)^{3} \Delta E_{2}\left\langle V P^{5} V P V\right\rangle$ 


\section{Continued}

\begin{tabular}{|c|c|c|}
\hline & 1 & $\left(\Delta E_{1}\right)^{2} \Delta E_{2} \Delta E_{1}\left\langle V P^{5} V P V\right\rangle$ \\
\hline & 1 & $\Delta E_{1} \Delta E_{2}\left(\Delta E_{1}\right)^{2}\left\langle V P^{5} V P V\right\rangle$ \\
\hline & 1 & $\Delta E_{2}\left(\Delta E_{1}\right)^{3}\left\langle V P^{5} V P V\right\rangle$ \\
\hline & 1 & $-\left(\Delta E_{1}\right)^{5}\left\langle V P^{6} V P V\right\rangle$ \\
\hline \multirow[t]{16}{*}{16} & 14 & $-\Delta E_{5}\left\langle V P V P^{2} V\right\rangle$ \\
\hline & 5 & $\Delta E_{1} \Delta E_{4}\left\langle V P V P^{3} V\right\rangle$ \\
\hline & 5 & $\Delta E_{4} \Delta E_{1}\left\langle V P V P^{3} V\right\rangle$ \\
\hline & 2 & $\Delta E_{3} \Delta E_{2}\left\langle V P V P^{3} V\right\rangle$ \\
\hline & 2 & $\Delta E_{2} \Delta E_{3}\left\langle V P V P^{3} V\right\rangle$ \\
\hline & 1 & $-\Delta E_{1}\left(\Delta E_{2}\right)^{2}\left\langle V P V P^{4} V\right\rangle$ \\
\hline & 1 & $-\Delta E_{2} \Delta E_{1} \Delta E_{2}\left\langle V P V P^{4} V\right\rangle$ \\
\hline & 1 & $-\left(\Delta E_{2}\right)^{2} \Delta E_{1}\left\langle V P V P^{4} V\right\rangle$ \\
\hline & 2 & $-\Delta E_{3}\left(\Delta E_{1}\right)^{2}\left\langle V P V P^{4} V\right\rangle$ \\
\hline & 2 & $-\Delta E_{1} \Delta E_{3} \Delta E_{1}\left\langle V P V P^{4} V\right\rangle$ \\
\hline & 2 & $-\left(\Delta E_{1}\right)^{2} \Delta E_{3}\left\langle V P V P^{4} V\right\rangle$ \\
\hline & 1 & $\left(\Delta E_{1}\right)^{3} \Delta E_{2}\left\langle V P V P^{5} V\right\rangle$ \\
\hline & 1 & $\left(\Delta E_{1}\right)^{2} \Delta E_{2} \Delta E_{1}\left\langle V P V P^{5} V\right\rangle$ \\
\hline & 1 & $\Delta E_{1} \Delta E_{2}\left(\Delta E_{1}\right)^{2}\left\langle V P V P^{5} V\right\rangle$ \\
\hline & 1 & $\Delta E_{2}\left(\Delta E_{1}\right)^{3}\left\langle V P V P^{5} V\right\rangle$ \\
\hline & 1 & $-\left(\Delta E_{1}\right)^{5}\left\langle V P V P^{6} V\right\rangle$ \\
\hline \multirow[t]{17}{*}{7} & 42 & $-\Delta E_{6}\left\langle V P^{2} V\right\rangle$ \\
\hline & 14 & $\Delta E_{1} \Delta E_{5}\left\langle V P^{3} V\right\rangle$ \\
\hline & 14 & $\Delta E_{5} \Delta E_{1}\left\langle V P^{3} V\right\rangle$ \\
\hline & 5 & $\Delta E_{4} \Delta E_{2}\left\langle V P^{3} V\right\rangle$ \\
\hline & $2 \times 2$ & $\Delta E_{3} \Delta E_{3}\left\langle V P^{3} V\right\rangle$ \\
\hline & 5 & $\Delta E_{2} \Delta E_{4}\left\langle V P^{3} V\right\rangle$ \\
\hline & 5 & $-\left(\Delta E_{1}\right)^{2} \Delta E_{4}\left\langle V P^{4} V\right\rangle$ \\
\hline & 5 & $-\Delta E_{4}\left(\Delta E_{1}\right)^{2}\left\langle V P^{4} V\right\rangle$ \\
\hline & 5 & $-\Delta E_{1} \Delta E_{4} \Delta E_{1}\left\langle V P^{4} V\right\rangle$ \\
\hline & 2 & $-\Delta E_{3} \Delta E_{2} \Delta E_{1}\left\langle V P^{4} V\right\rangle$ \\
\hline & 2 & $-\Delta E_{2} \Delta E_{3} \Delta E_{1}\left\langle V P^{4} V\right\rangle$ \\
\hline & 2 & $-\Delta E_{2} \Delta E_{1} \Delta E_{3}\left\langle V P^{4} V\right\rangle$ \\
\hline & 2 & $-\Delta E_{3} \Delta E_{1} \Delta E_{2}\left\langle V P^{4} V\right\rangle$ \\
\hline & 2 & $-\Delta E_{1} \Delta E_{3} \Delta E_{2}\left\langle V P^{4} V\right\rangle$ \\
\hline & 2 & $-\Delta E_{1} \Delta E_{2} \Delta E_{3}\left\langle V P^{4} V\right\rangle$ \\
\hline & 1 & $-\left(\Delta E_{2}\right)^{3}\left\langle V P^{4} V\right\rangle$ \\
\hline & 2 & $\Delta E_{3}\left(\Delta E_{1}\right)^{3}\left\langle V P^{5} V\right\rangle$ \\
\hline
\end{tabular}




$\begin{array}{cc}2 & \Delta E_{1} \Delta E_{3}\left(\Delta E_{1}\right)^{2}\left\langle V P^{5} V\right\rangle \\ 2 & \left(\Delta E_{1}\right)^{2} \Delta E_{3} \Delta E_{1}\left\langle V P^{5} V\right\rangle \\ 2 & \left(\Delta E_{1}\right)^{3} \Delta E_{3}\left\langle V P^{5} V\right\rangle \\ 1 & \left(\Delta E_{2}\right)^{2}\left(\Delta E_{1}\right)^{2}\left\langle V P^{5} V\right\rangle \\ 1 & \Delta E_{2} \Delta E_{1} \Delta E_{2} \Delta E_{1}\left\langle V P^{5} V\right\rangle \\ 1 & \Delta E_{2}\left(\Delta E_{1}\right)^{2} \Delta E_{2}\left\langle V P^{5} V\right\rangle \\ 1 & \Delta E_{1}\left(\Delta E_{2}\right)^{2} \Delta E_{1}\left\langle V P^{5} V\right\rangle \\ 1 & \Delta E_{1} \Delta E_{2} \Delta E_{1} \Delta E_{2}\left\langle V P^{5} V\right\rangle \\ 1 & \left(\Delta E_{1}\right)^{2}\left(\Delta E_{2}\right)^{2}\left\langle V P^{5} V\right\rangle \\ 1 & -\left(\Delta E_{1}\right)^{4} \Delta E_{2}\left\langle V P^{6} V\right\rangle \\ 1 & -\left(\Delta E_{1}\right)^{3} \Delta E_{2} \Delta E_{1}\left\langle V P^{6} V\right\rangle \\ 1 & -\left(\Delta E_{1}\right)^{2} \Delta E_{2}\left(\Delta E_{1}\right)^{2}\left\langle V P^{6} V\right\rangle \\ & -\Delta E_{1} \Delta E_{2}\left(\Delta E_{1}\right)^{3}\left\langle V P^{6} V\right\rangle \\ \sum_{p} S_{p}=21 \times 1+20 \times 2+3 \times 4+10 \times 5+3 \times 14+2 \times 10+2 \times 14+2 \times 42+132=429=S_{8} & -\Delta E_{2}\left(\Delta E_{1}\right)^{4}\left\langle V P^{6} V\right\rangle \\ 1 & \left(\Delta E_{1}\right)^{6}\left\langle V P^{7} V\right\rangle \\ & \end{array}$

not changed, as well as the Huby-Tong numbers presented in the central column. The only change will concern the terms entering the $\langle V, P\rangle$ brackets present on the right of the energy column. These bracket terms are coming from the main loop of time. In effect instead of terms

$$
\left\langle V P^{\alpha} V\right\rangle
$$

where

$$
2 \leq \alpha \leq 6
$$

is valid for $N=7$, we obtain in case of $N=8$ the bracket terms

$$
\left\langle V P^{\alpha} V P V\right\rangle
$$

for partition (93), and similar terms

$$
\left\langle V P V P^{\alpha} V\right\rangle
$$

for partition (94) entering Table 6.

The present Section can be concluded by saying that any partition number has its own set of energy terms. This property makes-to some extent-the considered partition number independent of other numbers of similar kind entering the partitions of $N-1$. The dependence of energy connected with some partition number on other partition numbers is due solely to the $\langle V, P\rangle$ term represented in brackets. This term is modified according to position which occupies a given partition number among other partition components which add together into the sum equal to $N-1$. The exponent of $P$ in the bracket terms denotes the number of the time loops which touch together in one contraction point. 
Table 7. Energy contributions given by the largest partition number due to $N=7$, i.e. $M=N-1=7-1=6$; see column one. The indices of the perturbation energies follow the partitions of $N-2=5$; see column two. The third column represents the $\mathrm{Hu}$ by-Tong number of the Schrödinger perturbation terms connected with the partitions given in column two. The sum of the number of perturbation terms entering the table is equal to $S_{6}$.

\begin{tabular}{|c|c|c|}
\hline Energy term & Partitions of $N-2=7-2=5$ & $\begin{array}{l}\text { The Huby-Tong number of } \\
\text { perturbation terms }\end{array}$ \\
\hline$-\Delta E_{5}\left\langle V P^{2} V\right\rangle$ & 5 & 14 \\
\hline$\Delta E_{1} \Delta E_{4}\left\langle V P^{3} V\right\rangle$ & 14 & 5 \\
\hline$\Delta E_{4} \Delta E_{1}\left\langle V P^{3} V\right\rangle$ & 41 & 5 \\
\hline$\Delta E_{3} \Delta E_{2}\left\langle V P^{3} V\right\rangle$ & 32 & 2 \\
\hline$\Delta E_{2} \Delta E_{3}\left\langle V P^{3} V\right\rangle$ & 23 & 2 \\
\hline$-\Delta E_{1}\left(\Delta E_{2}\right)^{2}\left\langle V P^{4} V\right\rangle$ & 122 & 1 \\
\hline$-\Delta E_{2} \Delta E_{1} \Delta E_{2}\left\langle V P^{4} V\right\rangle$ & 212 & 1 \\
\hline$-\left(\Delta E_{2}\right)^{2} \Delta E_{1}\left\langle V P^{4} V\right\rangle$ & 221 & 1 \\
\hline$-\Delta E_{3}\left(\Delta E_{1}\right)^{2}\left\langle V P^{4} V\right\rangle$ & 311 & 2 \\
\hline$-\Delta E_{1} \Delta E_{3} \Delta E_{1}\left\langle V P^{4} V\right\rangle$ & 131 & 2 \\
\hline$-\left(\Delta E_{1}\right)^{2} \Delta E_{3}\left\langle V P^{4} V\right\rangle$ & 113 & 2 \\
\hline$\left(\Delta E_{1}\right)^{3} \Delta E_{2}\left\langle V P^{5} V\right\rangle$ & 1112 & 1 \\
\hline$\left(\Delta E_{1}\right)^{2} \Delta E_{2} \Delta E_{1}\left\langle V P^{5} V\right\rangle$ & 1121 & 1 \\
\hline$\Delta E_{1} \Delta E_{2}\left(\Delta E_{1}\right)^{2}\left\langle V P^{5} V\right\rangle$ & 1211 & 1 \\
\hline$\Delta E_{2}\left(\Delta E_{1}\right)^{3}\left\langle V P^{5} V\right\rangle$ & 2111 & 1 \\
\hline \multirow[t]{2}{*}{$-\left(\Delta E_{1}\right)^{5}\left\langle V P^{6} V\right\rangle$} & 11111 & 1 \\
\hline & & $S_{6}=42$ \\
\hline
\end{tabular}

\section{Circular Scale of Time and Irreversibility of the Perturbation Process}

The effect of a perturbation of a quantum system can be roughly compared to a spoiling effect exerted on a toy: beyond of the original piece of the toy, many pieces from inside of it occur as a spoiling effect. Their size depends on the nature (construction) of the toy. It seems to be not reasonable to expect that the spoiling effect can be reversed in time. This means that a change of a quantum state due to perturbation is an irreversible effect. Some other examples of irreversibility are given in [18]. In fact we can do a transfer of the perturbed system into unperturbed one by removing the perturbation potential from the perturbed system. But the way in time due to such operation is different than the way from an originally unperturbed system to the perturbed one: just at the removing moment of the perturbation, the system is based on the perturbed eigenenergies and eigenstates. This means-in general-that the system reacts with the aid of the matrix elements between eigenstates and energies characteristic for 
the perturbed quantum state. The problem of comparison of two ways demands a precise knowledge of the time dependence of the system connected with an application of the perturbation in one case, and the time dependence connected with a removal of the perturbation in the reversed case.

\section{Summary of the Calculation Results}

Physically the perturbation problem is regarded as a set of collision events of a non-degenerate quantum system with a time-independent perturbation potential. The effect of collisions is expected to provide a definite change of energy of the original (unperturbed) system and transforms the system to another stationary (perturbed) state. The collisions process certainly occupies an interval of time which, however, does not enter the formalism.

A striking result is that a suitable choice of the sequence of collisions performed along a circular scale of time-dependent in practice only on the perturbation order $N$-does produce almost automatically the set of energy terms predicted by the Schrödinger perturbation theory. In this theory, as well as in its graphical modification due to Feynman, the corresponding calculations represent regularly a much more complicated task.

In the present case the method is based on an analysis of partitions concerning the number of time points present on the scale and contractions possible for these points. The contractions provide regularly the energy contributions of the order lower than considered $N$, but the total number of perturbation terms characteristic for a given $N$ becomes identical to that predicted by the number $S_{N}$ given by Huby and Tong [16] [17]; see (8) and for example the bottom of Table 6.

\section{General Remarks on Time and Its Scale Done from the Point of View of the Present Method}

So what is time? A short answer may begin with a statement that time is a parameter connected necessarily with a change: without a change the idea of time is hardly possible to be established. In classical physics the time concerns rather the geometry and not physical properties of a body. In fact the relation of time to geometry is a typical feature of the relativistic formalism. But the meaning of the time idea becomes much different in the quantum theory than classical one. Formally time has not its own operator and quanta of time cannot be treated in a way specified for other dynamical observables like, for example, the energy.

A well-known property of energy is its favourite position in the quantum theory. In practice, most accurate calculations done in that theory concern the energy. Simultaneously time is often qualified to be a quantity complementary to energy, but a real knowledge on time and ability to approach accurate data for it are in fact much poorer than in case of energy. In result time usually does not enter explicitly the quantum calculations but its effect is obtained-or rather approached-in a probabilistic, or statistical way. An exception is here perhaps a 
quantum counterpart of the classical Joule-Lenz law for a dissipated energy $\Delta E$ performed in course of the time interval $\Delta t$ [19] [20] [21]. For, when the problem concerns the transition of energy between two neighbouring quantum states, we have the relation

$$
\Delta E \Delta t=h
$$

where $h$ is the Planck constant. For $\Delta E$ in the atomic spectroscopy equal to about $1 \mathrm{eV}=1.6 \times 10^{-12} \mathrm{erg}$ and $h=6.67 \times 10^{-27} \mathrm{erg} \cdot \mathrm{sec}$, we obtain $\Delta t$ equal to about $10^{-15} \mathrm{sec}$. This interval is probably much too short to be investigated more deeply by both experimental and theoretical methods. The accuracy with which the relation (99) is satisfied increases with the quantum number $n$ possessed by the considered states.

The relation (99) can be extended to the treatment of energy transitions between more distant states than neighbouring ones [22], and results obtained for the emission intensity in this way, for example for the hydrogen atom [23] [24] [25], do not differ much from those calculated on the basis of a probabilistic quantum-mechanical formalism [26].

Another interesting property of time-more connected with the present paper-is its guiding property represented by its scale. Here the problem of the size of the time intervals is replaced by that of the sequence of events. Since centuries we are accustomed to the scale of time which does not allow for any repetition of the physical events. In fact it is assumed that everything in the nature follows this scale. This is the so-called straight-linear scale of time which means that the longer is the way travelled along this scale from a given situation or event, the farther is the time distance which separates this event or situation from the actual moment of time.

The aim of the present, and former papers of similar kind, is to show that such behaviour of time is not a general property characteristic for the quantum process. The point can be easily demonstrated on the example of the Schrödinger perturbation theory developed for a non-degenerate quantum state.

In its original form the Schrödinger perturbation theory did not apply the notion of time at all. In solving the algebraic equation for the perturbation energy which was a difference of energy between the perturbed and unperturbed state, only the unperturbed energies and matrix elements built up of the unperturbed quantum states and time-independent perturbation potential were taken into account. The number of terms necessary to be introduced into the equation, as well as the complication degree of these terms, increased rapidly with the perturbation order $N$.

Simultaneously none of a priori rule for construction of the individual terms entering the formalism could be obtained. This makes the Schrödinger perturbation treatment equivalent to a very tedious task.

Some decades after Schrödinger the Feynman diagrammatic method has been developed and applied to the same perturbation problem. But the method based on the conventional, i.e. straight-linear, scale of time became much ineffective 
for the perturbation purpose, especially for large $N$, mainly because of a drastic inflation of the number of the Feynman terms which had to be calculated and applied.

It became clear now that the Schrödinger perturbation problem could be essentially simplified when a circular scale of time-specified for each order $\mathrm{N}$-is developed. In the first step, the scale supplemented by the side loops of time due to contractions of the time points present on the main loop active for a given $N$, gave a one-to-one correspondence of the diagrams due to the active circular scale and Schrödinger perturbation terms. Moreover, the contributions due to the side loops of time obtained for a given $N$ could be combined into the Schrödinger perturbation energy terms having orders lower than $N$. This situation limited the number of new terms necessary to calculate when the examined order $N$ is increased to $N+1$ to a relatively small number of terms indicated by the main loop of time. In result it is found that a circular way of the arrangement of the collision events of an unperturbed system with the perturbation potential fits much better the calculation of the perturbed energy than an arrangement of the collision events along a straight-linear scale.

A final problem became the replacement of a rather inconvenient diagrammatic method of calculations by an algebraic approach. This has been done (see [8] [9] and the present paper) on the basis of a partition method applied to the number $M=N-1$. With the aid of partitions we can readily obtain the Schrödinger perturbation terms belonging to any $N$. This simplification is attained almost automatically due to the side loops of time present on each diagram for $N>2$.

The outlined result-which means that correction of the time scale leads to a proper and more compact expression for the energy-seems to be rather exceptional in quantum theory. A question may arise to which extent the scale applied for the perturbation problem can be of use for other problems.

Another question which can be formulated is: does the dependence of the quantum system on time-in specific boundary conditions-is an aimed, or aimless process? Quantum physics states solely-with no reference to time-that a non-perturbed quantum state may change into a perturbed one upon the action of an external potential, and the effect of this change on the system energy is known. Nevertheless also some interval of time is necessary for such change, but the size of that interval, as well as the time intervals of separate collisions of the system with the perturbation, remain unknown.

\section{Conclusions}

In principle we can have two kinds of the time scale. The first one is a continuous line, so any infinitesimally close point on the line is accessible from another point. In mechanics such kind of scale becomes to be considered as a time coordinate. For example we have a straight-linear coordinate extended from the minus to plus infinity; see Section 2. But another kind of the scale of time is also possible. Such scale reminds more the timetable of the train motion than a con- 
tinuous coordinate; see e.g. [9]. The most important feature of the scale is to register the sequence of events, for it becomes not so much important to represent the time distances between the events.

In fact the ordering effect possessed by the time scale of the second kind is found to be a sufficient property in constructing the Schrödinger perturbation series for energy, especially when this series concerns a non-degenerate quantum state.

A fundamental quality of the applied scale is that it is of a topologically circular shape. This means that collisions of the quantum state with the perturbation potential represent a cycle of collisions in which a given state $n$ can undergo first a transition from $n$ to state $p$, next a transition from $p$ to state $q$, etc., but a final state attained by transitions in a cycle is again the beginning state $n$. A suitable integration of the matrix elements representing the collision process over time gives the Schrödinger perturbation term characteristic for a given collisions cycle [6] [27]. The size of the cycle, therefore the number of collisions and variety of their effects, increases with the perturbation order $N$; see Equation (8). But when all contractions of the time points admissible for a given $N$ are taken into account, a full number of the Schrödinger perturbation terms characteristic for that $N$ are obtained. Some of these terms can be readily combined into the energy perturbation terms of the orders $N^{\prime}$ which are smaller than $N$.

Such a situation extremely facilitates the calculation of the Schrödinger perturbation series. Simultaneously, it demonstrates the power of a suitable choice of the time-ordering scale. The advantages of the scale are exhibited especially well when combined with partition properties of the perturbation order $N$.

\section{References}

[1] Berliner, A. and Scheel, K. (Eds.) (1932) Physikalisches Wörterbuch. Springer, Berlin. (In German)

[2] Schiff, L.I. (1968) Quantum Mechanics. 3rd Edition, McGraw-Hill, New York.

[3] Slater, J.C. (1960) Quantum Theory of the Atomic Structure Vol. 1. McGraw-Hill, New York.

[4] Olszewski, S. (1991) Zeitschrift für Naturforschung, 46A, 313.

[5] Olszewski, S. and Kwiatkowski, T. (1998) Computers \& Chemistry, 22, 445-461. https://doi.org/10.1016/S0097-8485(98)00023-0

[6] Olszewski, S. (2003) Trends in Physical Chemistry, 9, 69.

[7] Olszewski, S. (2013) Quantum Matter, 2, 481-483. https://doi.org/10.1166/qm.2013.1085

[8] Olszewski, S. (2014) Journal of Modern Physics, 5, 1502-1523. https://doi.org/10.4236/jmp.2014.515152

[9] Olszewski, S. (2014) Journal of Quantum Information Science, 4, 269-283. https://doi.org/10.4236/jqis.2014.44022

[10] Schrödinger, E. (1926) Annalen der Physik, 80, 437.

[11] Schrödinger, E. (1926) Annalen der Physik, 79, 361.

[12] Schrödinger, E. (1926) Annalen der Physik, 79, 489. 
[13] Schrödinger, E. (1926) Annalen der Physik, 81, 109.

[14] Mattuck, R.D. (1976) A Guide to Feynman Diagrams in a Many-Body Problem. 2nd Edition, McGraw-Hill, New York.

[15] Feynman, R.P. (1949) Physical Review, 76, 749. https://doi.org/10.1103/PhysRev.76.749

[16] Huby, R. (1961) Proceedings of the Physical Society(London), 78, 529. https://doi.org/10.1088/0370-1328/78/4/306

[17] Tong, B.Y. (1962) Proceedings of the Physical Society (London), 80, 1101. https://doi.org/10.1088/0370-1328/80/5/308

[18] Davis, P.C.W. (1994) Stirring up Trouble. In: Halliwell, J.J., Perez-Mercader, J. and Zurek, W.H., Eds., Physical Origins of Time Asymmetry, Cambridge University Press, Cambridge, 119-130.

[19] Olszewski, S. (2015) Journal of Modern Physics, 6, 1277-1288. https://doi.org/10.4236/jmp.2015.69133

[20] Olszewski, S. (2016) Reviews in Theoretical Science, 4,336-352. https://doi.org/10.1166/rits.2016.1066

[21] Olszewski, S. (2016) Journal of Modern Physics, 7, 162-174. https://doi.org/10.4236/jmp.2016.71018

[22] Olszewski, S. (2017) Journal of Modern Physics, 8, 1158-1174. https://doi.org/10.4236/jmp.2017.88077

[23] Olszewski, S. (2016) Journal of Modern Physics, 7, 827-851. https://doi.org/10.4236/jmp.2016.78076

[24] Olszewski, S. (2016) Journal of Modern Physics, 7, 1004-1020. https://doi.org/10.4236/jmp.2016.79091

[25] Olszewski, S. (2016) Journal of Modern Physics, 7, 1440-1448. https://doi.org/10.4236/jmp.2016.712131

[26] Condon, E.U. and Shortley, G.H. (1970) The Theory of Atomic Spectra. Cambridge University Press, Cambridge.

[27] Olszewski, S. (2015) Quantum Matter, 4, 523-532. https://doi.org/10.1166/qm.2015.1227

\section{Scientific Research Publishing}

Submit or recommend next manuscript to SCIRP and we will provide best service for you:

Accepting pre-submission inquiries through Email, Facebook, LinkedIn, Twitter, etc. A wide selection of journals (inclusive of 9 subjects, more than 200 journals) Providing 24-hour high-quality service User-friendly online submission system Fair and swift peer-review system Efficient typesetting and proofreading procedure Display of the result of downloads and visits, as well as the number of cited articles Maximum dissemination of your research work

Submit your manuscript at: http://papersubmission.scirp.org/ Or contact jmp@scirp.org 